\title{
Efficient Cooperative Protocols for Full-Duplex Relaying over Nakagami- $m$ Fading Channels
}

\author{
Mohammad Galal Khafagy, Member, IEEE, Amr Ismail, Member, IEEE, \\ Mohamed-Slim Alouini, Fellow, IEEE and Sonia Aïssa, Senior Member, IEEE
}

\begin{abstract}
In this work, efficient protocols are studied for full-duplex relaying (FDR) with loopback interference over Nakagami- $m$ block fading channels. Recently, a selective decodeand-forward (DF) protocol was proposed for FDR, and was shown to outperform existing protocols in terms of outage over Rayleigh-fading channels. In this work, we propose an incremental selective DF protocol that offers additional power savings, yet yields the same outage performance. We evaluate their outage performance over independent non-identically distributed Nakagami- $m$ fading links, and study their relative performance in terms of the signal-to-noise ratio cumulative distribution function via closed-form expressions. The offered diversity gain is also derived. In addition, we study their performance relative to their half-duplex counterparts, as well as known non-selective FDR protocols. We corroborate our theoretical results with simulation, and confirm that selective cooperation protocols outperform the known non-selective protocols in terms of outage. Finally, we show that depending on the loopback interference level, the proposed protocols can outperform their half-duplex counterparts when high spectral efficiencies are targeted.
\end{abstract}

Index Terms-Cooperative diversity, full-duplex relaying, incremental selective decode-and-forward, Nakagami- $m$ fading, self-interference, outage probability.

\section{INTRODUCTION}

$\mathbf{S}$ IGNIFICANT research efforts have been directed to multihop communications since Van Der Meulen's seminal work on three-terminal communication channels [1] and their capacity theorems due to Cover and El Gamal [2]. This is owing to their offered gains in terms of throughput enhancement and coverage extension. In multi-hop communications, intermediate relay nodes assist the communication of a sourcedestination pair by listening to the source transmissions and then forwarding the captured signal to the destination after the necessary processing. A cooperative relay can adopt one of two operation modes depending on how it regulates the simultaneity of its listening and forwarding phases, namely, half-duplex relaying (HDR) or full-duplex relaying (FDR). In HDR, relay listening and forwarding take place in two timeorthogonal phases, thus introducing a spectral-efficiency loss

M. G. Khafagy and M.-S. Alouini are with the Electrical Engineering Program, Computer, Electrical and Mathematical Sciences and Engineering (CEMSE) Division, King Abdullah University of Science and Technology (KAUST), Thuwal, Makkah Province, Saudi Arabia (email:\{mohammad.khafagy, slim.alouini\}@kaust.edu.sa).

A. Ismail was with the CEMSE Division, KAUST, Saudi Arabia. He is currently with the Telecommunications Research Laboratory, Toshiba Research Europe Limited, Bristol, UK (e-mail: amr.ismail@toshiba-trel.com).

S. Aïssa is with the Institut National de la Recherche Scientifique (INRS), University of Quebec, Montreal, QC, Canada (e-mail: aissa@emt.inrs.ca).

This work was supported in part by King Abdullah University of Science and Technology (KAUST), Saudi Arabia, and in part by King Abdulaziz City for Science and Technology (KACST), Saudi Arabia. The statements made herein are solely the responsibility of the authors. when compared to direct transmission. On the other hand, FDR supports simultaneous listening/forwarding phases over the same channel resource, and hence it avoids the spectralefficiency loss in HDR.

\section{A. Related Work}

Although FDR eliminates the known prelog factor from the capacity expressions of relay channels [3], performance can be significantly degraded in practice from a different perspective. Indeed, since the relay transmits and receives over the same channel, an interference link, called loopback or echo interference link, is introduced from the relay transmitter to its receiver. Currently, all isolation and cancellation techniques for FDR, see [4]-[9] and the references therein, cannot perfectly prevent relay transmissions from leaking towards its receiver and causing undesirable interference. As a result, a level of residual self-interference (RSI) persists and adversely affects the effective signal-to-noise ratio (SNR) inside the capacity logarithm. Thus, increasing the relay power does not necessarily boost the end-to-end performance, which defines one challenge in FDR. Another challenge in FDR lies in leveraging the existing cooperative diversity due to the message replicas received at the destination from the source and the relay(s).

Recent work on FDR with RSI adopted instant decoding at the destination under Rayleigh [7]-[9] and Nakagami-m [10], [11] block fading, where each received codeword is separately processed. Since the relay forwards a delayed version of the source signal, this decoding approach could not exploit the available diversity, and the direct source transmissions were treated as noise to the stronger relay signal. In an attempt to exploit this channel diversity, the effect of RSI was introduced in [12], [13] to the block Markov superposition coding protocol in [14], and the outage performance was evaluated for Rayleigh and Nakagami- $m$ fading scenarios, respectively. However, since the RSI can severely degrade the performance over the source-to-destination multi-hop path, fixed relaying based on superposition coding can still fall short in attaining the best outage performance in FDR channels with RSI.

In an attempt to simultaneously tackle the above challenges, cooperative protocols that were proposed earlier in the HDR literature are revisited. In [15], Laneman et al. proposed several HDR protocols and studied their outage performance. Among these protocols is the selective decode-and-forward (SDF) protocol in which the relay assists only when the source-relay link is not in outage. Thus, SDF avoids as much as possible forwarding mere interference to the destination, giving the latter a chance to recover the message from the direct link. Also proposed in [15] is the incremental decodeand-forward (IDF) protocol where the relay only assists upon 
the reception of a one-bit feedback from the destination declaring an outage in the direct source-destination link. This protocol was proposed to primarily alleviate the inherent rate loss in HDR by allowing the destination to solely rely on the direct link as long as it is in a good condition, and hence it avoids the dedication of a time slot for relay forwarding.

In recent work [16], SDF was proposed for employment in FDR where it was shown to outperform existing schemes in terms of outage in block Rayleigh-fading environments. Since relay transmissions directly affect the channel outage state in decode-and-forward relaying, selecting when to cooperate is crucial for the end-to-end performance. Specifically, when the relay finds the source-relay link in outage due to its transmissions, it is better to go into non-cooperative mode. This helps in two directions, namely, saving the relay power, and giving a chance for the destination to attempt decoding from the direct source signal without unnecessary relay interference. In [12], the HDR versions of SDF and IDF cooperation were also compared to non-selective FDR protocols over Rayleigh-fading channels, and it was shown that selective and incremental HDR protocols can outperform nonselective FDR. This further motivates our study to investigate the relative performance of selective FDR protocols to their HDR counterparts.

\section{B. Contribution}

Our contribution in this work can be summarized as follows:

- The outage analysis of SDF-FDR is generalized to Nakagami- $m$ fading with integer $m$ parameters, subsuming the Rayleigh-fading result in [16] as a special case.

- Motivated by the IDF protocol in [15], we propose a novel FDR protocol which we call incremental selective decode-and-forward FDR (ISDF-FDR). In addition to the condition on the source-relay link outage, ISDF-FDR demands a one-bit feedback from the destination declaring an outage in the direct link to proceed with cooperation. This protocol offers the same outage performance of SDF-FDR, yet yields higher relay power savings. ${ }^{1}$

- To capture the performance difference between SDF-FDR and ISDF-FDR, we derive closed-form expressions for the cumulative distribution function (CDF) of their endto-end SNR.

- Moreover, we study the performance of these two protocols relative to their HDR counterparts, in addition to other known non-selective FDR schemes.

- We derive and compare the diversity order of the selective cooperative protocols under consideration to their nonselective counterparts under Rayleigh fading. We show that the studied selective protocols get around the known error floor phenomenon in FDR by providing an additional diversity via exploiting the direct source-destination link.

\footnotetext{
${ }^{1}$ While this work was in progress, preliminary results for the Rayleighfading scenario were accepted for presentation in IEEE GLOBECOM'13 [17]. The currently presented work reports the full results for general Nakagami- $m$ fading with integer $m$ parameters which subsumes the results in [17] as a special case when $m=1$. The presented simulation results and comparisons are also novel, where recently analyzed FDR protocols as well as their HDR counterparts are compared to the proposed ones.
}

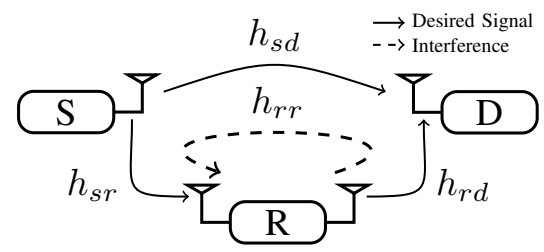

Fig. 1. Cooperative communication system with full-duplex relaying.

- We corroborate our theoretical results with simulations, and show the relative relay power savings in comparison to non-selective cooperation in which the relay cooperates regardless of channel conditions.

\section{Paper Outline}

The rest of the paper is organized as follows. In Section II, we explain the system model and the main notations adopted in the paper. We introduce the cooperation policies of the two selective relaying protocols under study in Section III, and analyze their outage performance in closed-form. In section IV, we derive closed-form expressions for the CDF of the end-to-end SNR for both protocols to capture their relative performance. A summary of the FDR and HDR cooperation protocols that are considered for performance comparison is discussed in Section V. Numerical simulations are performed and their results are reported in Section VI to validate our analytical results. Finally, we conclude our findings in Section VII. Relevant proofs are deferred to the appendices.

\section{SySTEM MOdeL}

\section{A. Channel Model}

We consider the relay channel depicted in Fig. 1, where a source (S) communicates with a destination (D) through a direct link with a possibly non-negligible gain. A relay (R) can assist the S-D communication via a regenerative decodeand-forward (DF) approach. We denote the channel between node $i \in\{s, r\}$ and node $j \in\{r, d\}$ by $h_{i j}$, and assume that it experiences block fading. Specifically, $h_{i j}$ remains constant over one channel block, and varies independently from one block to another following a Nakagami- $m$ fading model with shape parameter $m_{i j}$ and average power $\mathbb{E}\left\{\left|h_{i j}\right|^{2}\right\}=\pi_{i j}$, [18], [19]. Accordingly, the channel gain, $\left|h_{i j}\right|^{2}$, is a Gamma random variable (RV) with shape parameter $m_{i j}$ and scale parameter $\theta_{i j}=\frac{\pi_{i j}}{m_{i j}}$, for which we use the shorthand notation $\mathcal{G}\left(m_{i j}, \theta_{i j}\right)$. It should be noted that for a Gamma RV $X \sim$ $\mathcal{G}(m, \theta)$, its probability density function (PDF) and CDF are respectively given by

$f_{X}(x ; m, \theta)=\frac{x^{m-1} e^{-\frac{x}{\theta}}}{\Gamma(m) \theta^{m}}$ and $F_{X}(x ; m, \theta)=\frac{\gamma\left(m, \frac{x}{\theta}\right)}{\Gamma(m)}$,

where $\gamma(a, b)=\int_{0}^{b} t^{a-1} e^{-t} d t$ denotes the lower incomplete Gamma function, while $\Gamma(a)$ denotes the Gamma function [20]. The Nakagami- $m$ fading model spans a wide range of fading scenarios that subsumes Rayleigh fading as a special case when $m=1$. Also, for $m>1$, a one-to-one mapping exists between its $m$ parameter and the Rician $K$ factor, which allows it to closely approximate the Rician distribution and accordingly capture line-of-sight effects [19]. For notational convenience, we assume that $h_{r r}$ denotes the RSI channel 


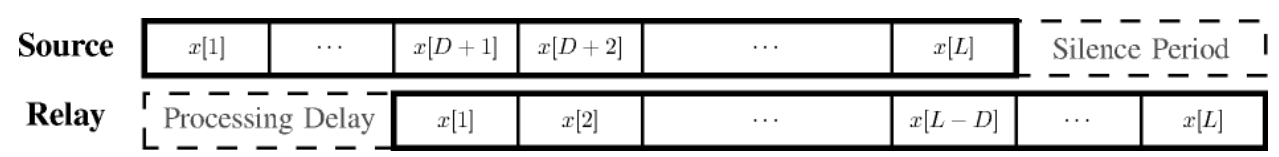

Fig. 2. Block transmission scheme, where $\boldsymbol{x}[t], t \in\{1,2, \cdots, L\}$, is the $t^{t h} n$-dimensional codeword transmitted by the source.

after undergoing all known practical isolation and cancellation techniques (see [5]-[9] and the references therein). Also, without loss of generality, all additive white Gaussian noise components are assumed of unit variance.

\section{B. Notation}

Throughout the rest of the paper, we use $x, \boldsymbol{x}$ and $\boldsymbol{X}$ to respectively denote a scalar quantity, a column vector, and a matrix. Also, we use $(.)^{T}$ to denote a transposition operation, while $(.)^{H}$ denotes transposition with complex conjugation. The determinant of a matrix $\boldsymbol{X}$ is denoted by $\operatorname{det}\{\boldsymbol{X}\}$. We use $\mathbb{P}\{A\}$ to denote the probability of occurrence of an event $A$, while $\mathbb{E}\{$.$\} is used to denote the statistical expectation. We$ use $\Gamma_{i j}$ to denote the random variable of the received SNR via the link $h_{i j}$ taking transmit power into account, with $\gamma_{i j}$ being a specific realization of it. Also, $f_{i j}(x), F_{i j}(x)$ and $\bar{F}_{i j}(x)$ are used to respectively denote the PDF, CDF and complementary cumulative distribution function $(\mathrm{CCDF})$ of $\Gamma_{i j}$. Moreover, $f_{i j}(x \mid A), F_{i j}(x \mid A)$ and $\bar{F}_{i j}(x \mid A)$ respectively denote its PDF, $\mathrm{CDF}$ and CCDF conditioned on the occurrence of event $A$. Assuming Gaussian channel inputs and unit bandwidth, $C_{i j}$ is used to denote the information capacity in bits per channel use (bpcu) for the link $h_{i j}$, i.e., $\log _{2}\left(1+\Gamma_{i j}\right)$, while $\mathcal{O}_{i j}$ and $\overline{\mathcal{O}}_{i j}$ respectively denote its outage event, i.e., $C_{i j}<R$ or equivalently $\Gamma_{i j}<\gamma_{t h}=2^{R}-1$, and its complement. Also, $\mathcal{P}_{i j}$ denotes the outage probability in the link $h_{i j}$, i.e., $\mathcal{P}_{i j}=\mathbb{P}\left\{\mathcal{O}_{i j}\right\}=F_{i j}\left(\gamma_{t h}\right)$, while $\overline{\mathcal{P}}_{i j}=1-\mathcal{P}_{i j}$.

\section{Signal Model}

We consider the block transmission scheme in Fig. 2. As shown, communication takes place over one super-block, which is composed of $L+D$ blocks. All channel gains are assumed to remain constant over the entire super-block. Defining $R$ as the fixed transmission rate and $n$ as the block size, the source node $\mathrm{S}$ intends to convey, at block $t \in\{1,2, \cdots, L\}$, a message $W[t] \in \mathcal{M}=\left\{1,2, \cdots, 2^{n R}\right\}$ to the destination node $\mathrm{D}$. At the source, an encoding function $\mathcal{E}: \mathcal{M} \rightarrow \mathcal{C}$ is applied to the message $W[t]$ to yield an $n$-dimensional codeword $\boldsymbol{x}[t] \in \mathcal{C}$, where $\mathcal{C}$ is the source codebook with cardinality $|\mathcal{C}|=|\mathcal{M}|=2^{n R}$ and whose rate $R$ is expressed in terms of SNR parameter, $\gamma_{t h}$, as $\log _{2}\left(1+\gamma_{t h}\right)$. Note that each block spans $n$ symbol durations due to the transmission of $n$-dimensional codewords. We assume that $n$ is fairly large, thereby validating the use of information theoretic tools.

It is assumed that the outage event dominates the error event. Thus, for a node to be able to attempt decoding the source message, its received SNR should exceed the threshold $\gamma_{t h}=2^{R}-1$. In the considered selective relaying protocols, certain channel conditions should be satisfied in order to trigger the relay to cooperate. These conditions should at least guarantee that no outage occurs in the S-R link. That is, when the relay enters the cooperative mode, it regenerates the successfully decoded source message taking its processing delay into account. In what follows, we discuss the signal model with and without relay assistance.

1) Cooperative Mode: Taking the relay processing delay of $D$ blocks into account, the relay simultaneously forwards $\boldsymbol{x}[t-$ $D]$, which imposes self-interference. Thus, the $n$-dimensional received signal at node $\mathrm{R}$ at block $t$ is given by

$$
\boldsymbol{y}_{r}[t]=h_{s r} \boldsymbol{x}[t]+h_{r r} \boldsymbol{x}[t-D]+\boldsymbol{n}_{r}[t],
$$

where $\boldsymbol{n}_{r}[t] \in \mathbb{C}^{n \times 1}$ denotes the relay noise. Without loss of generality, we assume that each symbol in $\boldsymbol{x}[t]$ has an average power of unity, and that the transmit powers of the source and the relay are absorbed into the channel coefficients for ease of notation. It should be noted that, if a Gamma $\mathrm{RV} X \sim$ $\mathcal{G}(m, \theta)$ is scaled by a constant $\alpha$, then $Y=\alpha X \sim \mathcal{G}(m, \alpha \theta)$. Thus, transmit power will only affect the scale parameter of the Gamma distributed channel gain. Hence, the received signal at the destination is given by

$$
\boldsymbol{y}_{d}[t]=h_{s d} \boldsymbol{x}[t]+h_{r d} \boldsymbol{x}[t-D]+\boldsymbol{n}_{d}[t],
$$

where $\boldsymbol{n}_{d}[t] \in \mathbb{C}^{n \times 1}$ denotes the destination noise at block $t$. We rewrite (3) in vector form to jointly account for the $L+D$ signals received over the entire super-block as

$$
\begin{gathered}
\boldsymbol{y}_{d}=\boldsymbol{H} \boldsymbol{x}+\boldsymbol{n}_{d}, \\
\text { where } \left.\boldsymbol{y}_{d}=\boldsymbol{y}_{d}[1]^{T}, \ldots, \boldsymbol{y}_{d}[L+D]^{T}\right)^{T}, \quad \boldsymbol{x}= \\
\left(\boldsymbol{x}[1]^{T}, \ldots, \boldsymbol{x}[L]^{T}\right)^{T}, \boldsymbol{n}_{d}=\left(\boldsymbol{n}_{d}[1]^{T}, \ldots, \boldsymbol{n}_{d}[L+D]^{T}\right)^{T} \text { and } \\
\left.\qquad \begin{array}{c}
\boldsymbol{I}_{n L} \\
\mathbf{0}_{n D \times n L}
\end{array}\right]+h_{r d}\left[\begin{array}{c}
\mathbf{0}_{n D \times n L} \\
\boldsymbol{I}_{n L}
\end{array}\right] .
\end{gathered}
$$

2) Non-Cooperative Mode: In this case, the relay does not assist. Thus, the received signal at the destination at block $t$ is given by

$$
\boldsymbol{y}_{d}[t]=h_{s d} \boldsymbol{x}[t]+\boldsymbol{n}_{d}[t] .
$$

For large $\frac{L}{D}$, in order to keep the same block structure adopted in the no-outage case, it is equivalent to use the vector form in (4) with the value of $\theta_{r d}$ set to zero.

\section{Instantaneous Link Capacities}

To analyze the end-to-end channel outage, the instantaneous capacities of the individual links need to be first provided. Assuming complex Gaussian channel inputs and unit bandwidth, the information capacity of link $h_{i j}$ is given by $\mathcal{C}_{i j}=\log _{2}\left(1+\Gamma_{i j}\right)$ bpcu, where $\Gamma_{s d}=\left|h_{s d}\right|^{2}$ and $\Gamma_{r d}=\left|h_{r d}\right|^{2}$, while $\Gamma_{s r}=\frac{\left|h_{s r}\right|^{2}}{\left|h_{r r}\right|^{2}+1}$ denotes the signal-tointerference-plus-noise ratio (SINR) in the S-R link with the RSI effect taken into account as an additional noise term.

At the destination, we use $C_{(s, r) \rightarrow d}$ to denote the information capacity per super-block in the virtual multiple-input single-output (MISO) channel formed by $\mathrm{S}$ and $\mathrm{R}$ as the transmitter side and by $\mathrm{D}$ as the receiver side. It is worth mentioning that no channel state information is assumed at the transmitter side, and hence, no power or rate adaptation is 
possible. Again, assuming complex Gaussian inputs and unit bandwidth, it is readily given by

$C_{(s, r) \rightarrow d}=\log _{2} \operatorname{det}\left\{\boldsymbol{I}_{n L}+\boldsymbol{H}^{H} \boldsymbol{H}\right\}=\log _{2} \prod_{i=1}^{n L}\left(1+\lambda_{i}\right)$,

where

$$
\boldsymbol{H}^{H} \boldsymbol{H}=\alpha \boldsymbol{I}_{n L}+\beta \boldsymbol{B}_{n L}{ }^{n D}+\beta^{*} \boldsymbol{F}_{n L}{ }^{n D},
$$

with $\alpha=\left|h_{s d}\right|^{2}+\left|h_{r d}\right|^{2}=\Gamma_{s d}+\Gamma_{r d}$ and $\beta=h_{s d}^{*} h_{r d}$, while $\boldsymbol{B}_{\ell}\left(\boldsymbol{F}_{\ell}\right)$ denotes a square backward (forward) shift matrix of size $\ell$, with ones only on the first subdiagonal (superdiagonal) and zeros elsewhere. Also, $\left\{\lambda_{i}\right\}_{i=1}^{n L}$ denote the $n L$ eigenvalues of $\boldsymbol{H}^{H} \boldsymbol{H}$. For the special case of $D=n=1, \boldsymbol{H}^{H} \boldsymbol{H}$ is an $L \times L$ tridiagonal Toeplitz matrix, whose $L$ eigenvalues are known in closed-form, [21, pp. 80], as

$$
\lambda_{i}=\alpha+2|\beta| \cos \frac{i \pi}{L+1}, i \in\{1,2, \cdots, L\} .
$$

We show in Appendix A that for $L=k D$ with $k \in \mathbb{Z}^{+}$, and for general $n \in \mathbb{Z}^{+}$, the $n L$ eigenvalues are generalized to:

$\lambda_{n D(i-1)+1: n D i}=\alpha+2|\beta| \cos \frac{i D \pi}{L+D}, i \in\{1,2, \cdots, k\},(10)$ where $\lambda_{i: j}$ denotes the set of eigenvalues $\left\{\lambda_{i}, \lambda_{i+1}, \cdots, \lambda_{j}\right\}$.

\section{OUtage Probability}

Before we proceed further, and since functions of Gamma $\mathrm{RV}$ s will be frequently incurred, it is convenient to start with stating two remarks about the distribution of such functions which will be used throughout the analysis to follow.

Remark 1 (CDF of $S=X_{1}+X_{2}$ ): For two independent Gamma RVs, $X_{1} \sim \mathcal{G}\left(m_{1}, \theta_{1}\right)$ and $X_{2} \sim \mathcal{G}\left(m_{2}, \theta_{2}\right)$, with integer shape parameters and possibly distinct scale parameters, $S=X_{1}+X_{2}$ has the following CDF [22]:

$F_{S}(s ; \boldsymbol{p})=\frac{\gamma\left(m_{2}, \frac{s}{\theta_{2}}\right)}{\Gamma\left(m_{2}\right)}-\sum_{k=0}^{m_{1}-1} \frac{A}{\Gamma(v)}\left(\frac{s}{\theta_{1}}\right)^{k}{ }_{1} F_{1}(u ; v ; w)$,

where $\boldsymbol{p}=\left(m_{1}, \theta_{1}, m_{2}, \theta_{2}\right)$ is a vector holding the shape and scale parameters, $A=\left(\frac{s}{\theta_{2}}\right)^{m_{2}} \exp \left(-\frac{s}{\theta_{2}}\right), u=k+1, v=$ $m_{2}+k+1, w=\frac{\theta_{1}-\theta_{2}}{\theta_{1} \theta_{2}} s$ and ${ }_{1} F_{1}(u ; v ; w)$ is the Kummer's confluent hypergeometric function [20, Eq. 13.1.2].

Remark 2 (CDF of $\left.Z=\frac{X_{1}}{X_{2}+1}\right)$ : For two independent Gamma RVs, $X_{1} \sim \mathcal{G}\left(m_{1}, \theta_{1}\right)$ and $X_{2} \sim \mathcal{G}\left(m_{2}, \theta_{2}\right)$, with integer shape parameters and possibly distinct scale parameters, $Z=\frac{X_{1}}{X_{2}+1}$ has the following CDF [10], [13]:

$$
F_{Z}(z ; \boldsymbol{p})=\frac{\gamma\left(m_{1}, \frac{z}{\theta_{1}}\right)}{\Gamma\left(m_{1}\right)}+B \sum_{k=0}^{m_{2}-1} \frac{c^{-d}}{\theta_{2}{ }^{k}} W_{a, b}[c],
$$

where $W_{a, b}[c]$ is the Whittaker function [20, Eq. 13.1.33], $a=\frac{m_{1}-k-1}{2}, b=\frac{-m_{1}-k}{2}, c=\frac{z}{\theta_{1}}+\frac{1}{\theta_{2}}, d=\frac{m_{1}+k+1}{2}$ and

$$
B=\frac{\exp \left(-\frac{1}{2}\left(\frac{z}{\theta_{1}}-\frac{1}{\theta_{2}}\right)\right)}{\Gamma\left(m_{1}\right)}\left(\frac{z}{\theta_{1}}\right)^{m_{1}} .
$$

Since the SNRs $\Gamma_{s d}$ and $\Gamma_{r d}$ are respectively $\mathcal{G}\left(m_{s d}, \theta_{s d}\right)$ and $\mathcal{G}\left(m_{r d}, \theta_{r d}\right)$ distributed, it follows from (1) that for $i \in$ $\{s, r\}$ the SNR CDF is given by

$$
F_{i d}\left(\gamma_{i d}\right)=F_{X}\left(\gamma_{i d} ; m_{i d}, \theta_{i d}\right) .
$$

Also, since $\Gamma_{s r}=\frac{\left|h_{s r}\right|^{2}}{\left|h_{r r}\right|^{2}+1}$, it follows from (12) that the CDF of the received SNR via the S-R link is given by

$$
F_{s r}\left(\gamma_{s r}\right)=F_{Z}\left(\gamma_{s r} ; \boldsymbol{p}_{1}\right)
$$

with $\boldsymbol{p}_{1}=\left(m_{s r}, \theta_{s r}, m_{r r}, \theta_{r r}\right)$. In what follows, we proceed with the cooperation protocols and their outage performance.

\section{A. Selective DF-FDR (SDF-FDR) Cooperation}

We briefly explain the cooperation policy of SDF-FDR as proposed in [16], and generalize its outage performance to Nakagami- $m$ fading. In SDF-FDR, cooperation takes place only when the S-R link is not in outage. An outage is declared when one of two events occurs: 1) the S-R link is in outage, hence no cooperation takes place, while the S-D link goes into an outage state, or 2) the S-R link is not in outage, thus relay assistance is available, but an outage occurs in the MISO channel. This is more formally defined as given in [16] by

$$
\mathcal{P}_{S D F}=\mathcal{P}_{s r} \mathcal{P}_{s d}+\overline{\mathcal{P}}_{s r} \mathcal{P}_{(s, r) \rightarrow d} \text {. }
$$

where $\mathcal{P}_{s d}$ is nothing but the SNR CDF of the S-D link evaluated at $\gamma_{t h}$, i.e.,

$$
\mathcal{P}_{s d}=\mathbb{P}\left\{\Gamma_{s d}<\gamma_{t h}\right\}=F_{s d}\left(\gamma_{t h}\right) .
$$

Similarly,

$$
\mathcal{P}_{s r}=\mathbb{P}\left\{\Gamma_{s r}<\gamma_{t h}\right\}=F_{s r}\left(\gamma_{t h}\right) .
$$

In (16), $\mathcal{P}_{(s, r) \rightarrow d}$ is the probability of outage in the virtual MISO channel formed by $\mathrm{S}$ and $\mathrm{R}$ as the transmitter side and by $\mathrm{D}$ as the receiver side. Noting that $C_{(s, r) \rightarrow d}$ denotes the information capacity per super-block, $\mathcal{P}_{(s, r) \rightarrow d}$ is defined as

$$
\mathcal{P}_{(s, r) \rightarrow d}=\mathbb{P}\left\{\frac{C_{(s, r) \rightarrow d}}{n(L+D)}<\frac{n L}{n(L+D)} R\right\},
$$

where the factor $\frac{n L}{n(L+D)}$ accounts for the fact that the source only transmits in $L$ out of $L+D$ blocks. In [16], it was shown using (7)-(10) to be well-approximated by

$$
\mathcal{P}_{(s, r) \rightarrow d} \approx \mathbb{P}\left\{\alpha \leq \gamma_{t h}\right\} .
$$

Since $\Gamma_{s d}$ and $\Gamma_{r d}$ are independent and respectively distributed as $\mathcal{G}\left(m_{s d}, \theta_{s d}\right)$ and $\mathcal{G}\left(m_{r d}, \theta_{r d}\right), \mathcal{P}_{(s, r) \rightarrow d}$ is then given for the Nakagami- $m$ fading scenario via (11) as

$$
\mathcal{P}_{(s, r) \rightarrow d} \approx F_{S}\left(\gamma_{t h} ; \boldsymbol{p}_{2}\right)
$$

with $\boldsymbol{p}_{2}=\left(m_{r d}, \theta_{r d}, m_{s d}, \theta_{s d}\right)$. More generally, it follows from [16] and (11) that the CDF of the SNR in the MISO channel, denoted as $F_{(s, r) \rightarrow d}(\gamma)$, is approximately given as

$$
F_{(s, r) \rightarrow d}(\gamma) \approx \mathbb{P}\{\alpha \leq \gamma\}=F_{S}\left(\gamma ; \boldsymbol{p}_{2}\right) .
$$

This interesting result means that the MISO channel can be equivalently viewed as a point-to-point single-antenna channel with an effective SNR $\Gamma_{\text {eff }} \approx \alpha=\Gamma_{s d}+\Gamma_{r d}$. Therefore, according to the SDF-FDR cooperation policy, the effective SNR at the destination node, denoted by $\Gamma$, can be approximately given by

$$
\Gamma \approx \begin{cases}\Gamma_{s d}, & \mathcal{O}_{s r} \\ \Gamma_{s d}+\Gamma_{r d}, & \overline{\mathcal{O}}_{s r}\end{cases}
$$

\section{B. Incremental Selective DF-FDR (ISDF-FDR) Cooperation}

Here, we propose another protocol that seeks further relay power savings. We call this protocol as incremental selective DF-FDR (ISDF-FDR). The selective part comes from the same relay selectivity in forwarding the source message depending on the outage state of the S-R link. However, when outage performance is our main concern, the relay does not need to 


$$
\begin{aligned}
& F_{1}(\gamma)=\frac{F_{X}\left(\gamma ; m_{s d}, \theta_{s d}\right)}{\mathcal{P}_{s d}}-\sum_{m=0}^{m_{r d}-1} \frac{\gamma^{m_{s d}+m} e^{-\frac{\gamma}{\theta_{r d}}}{ }_{1} F_{1}\left(m_{s d} ; m_{s d}+m+1 ;\left(\frac{1}{\theta_{r d}}-\frac{1}{\theta_{s d}}\right) \gamma\right)}{\mathcal{P}_{s d} \Gamma\left(m_{s d}+m+1\right) \theta_{r d}{ }^{m} \theta_{s d}{ }_{s d}}=\frac{F_{S}\left(\gamma ; \boldsymbol{p}_{2}\right)}{\mathcal{P}_{s d}} \\
& F_{2}(\gamma)=1-\sum_{m=0}^{m_{r d}-1} \sum_{k=0}^{m} \frac{\left(\gamma-\gamma_{t h}\right)^{m-k} \gamma_{t h}^{m_{s d}+k} e^{-\frac{\gamma}{\theta_{r d}}}{ }_{1} F_{1}\left(m_{s d} ; m_{s d}+k+1 ;\left(\frac{1}{\theta_{r d}}-\frac{1}{\theta_{s d}}\right) \gamma_{t h}\right)}{\mathcal{P}_{s d} \Gamma\left(m_{s d}+k+1\right) \Gamma(m-k+1) \theta_{r d}{ }^{m} \theta_{s d} m_{s d}}
\end{aligned}
$$

always forward when it successfully decodes. Instead, relay assistance becomes necessary only upon the reception of a one-bit feedback from the destination at the beginning of the super-block declaring an outage and asking for assistance. Therefore, an outage occurs in the channel under consideration when one of two events occurs: 1) the S-D link goes in outage, while the S-R link is in outage. In this case, the relay is unable to assist and outage occurs with probability $\mathcal{P}_{s r} \mathcal{P}_{s d}$ due to the independence of channel fading coefficients, or 2) the S-D link goes in outage while the S-R link is not in outage, but the cooperative MISO channel is in outage. The latter outage event represents an intersection of three events. We know that the event of no outage in the S-R link is independent of the S-D and the MISO channel outage events. Also, we know that cooperation cannot decrease the mutual information, and thus, the outage capacity of the MISO channel is at least equal to that of the S-D link. Hence, the intersection of the outage events in the S-D and the MISO channel is the outage event in the MISO channel itself. Therefore, the probability of this event is equal to $\overline{\mathcal{P}}_{s r} \mathcal{P}_{(s, r) \rightarrow d}$. As such, the outage probability in the channel under consideration is given by

$$
\mathcal{P}_{I S D F}=\mathcal{P}_{s r} \mathcal{P}_{s d}+\overline{\mathcal{P}}_{s r} \mathcal{P}_{(s, r) \rightarrow d}
$$

while the received SNR profile is approximately given by

$$
\Gamma \approx \begin{cases}\Gamma_{s d}, & \overline{\mathcal{O}}_{s d} \cup\left(\mathcal{O}_{s r} \cap \mathcal{O}_{s d}\right), \\ \Gamma_{s d}+\Gamma_{r d}, & \overline{\mathcal{O}}_{s r} \cap \mathcal{O}_{s d} .\end{cases}
$$

Clearly, (24) is exactly equal to that of the SDF protocol given in (16). Although SDF and ISDF have different cooperation policies, and accordingly different SNR profiles as given in (23) and (25), they do have exactly the same outage events, and hence their outage performance overlaps. In other words, as it can be noticed from (23) and (25), the SDF protocol offers extra relay cooperation in the particular non-outage event of $\overline{\mathcal{O}}_{s r} \cap \overline{\mathcal{O}}_{s d}$ that does not matter in terms of the outage performance. Since we can easily expect that SDF can offer higher performance beyond the outage metric due to the more cooperation it offers, outage analysis does not suffice to capture and distinguish the relative performance of both protocols.

\section{SNR PERFORMANCE}

Due to the selective nature of the adopted cooperation policies, the received SNR at the destination and its probability distribution varies depending on the channel outage states. Now, we analyze the end-to-end SNR performance of each of the two protocols.

\section{A. ISDF-FDR}

Let $\left\{A_{i}\right\}_{i=1}^{4}$ denote the intersection of outage events which are defined, along with their probabilities, as follows:

$$
\begin{array}{ll}
A_{1} \triangleq \mathcal{O}_{s r} \cap \mathcal{O}_{s d}, & \mathbb{P}\left\{A_{1}\right\}=\mathcal{P}_{s r} \mathcal{P}_{s d}, \\
A_{2} \triangleq \overline{\mathcal{O}}_{s r} \cap \mathcal{O}_{s d}, & \mathbb{P}\left\{A_{2}\right\}=\overline{\mathcal{P}}_{s r} \mathcal{P}_{s d}, \\
A_{3} \triangleq \mathcal{O}_{s r} \cap \overline{\mathcal{O}}_{s d}, & \mathbb{P}\left\{A_{3}\right\}=\mathcal{P}_{s r} \overline{\mathcal{P}}_{s d}, \\
A_{4} \triangleq \overline{\mathcal{O}}_{s r} \cap \overline{\mathcal{O}}_{s d}, & \mathbb{P}\left\{A_{4}\right\}=\overline{\mathcal{P}}_{s r} \overline{\mathcal{P}}_{s d} .
\end{array}
$$

Clearly, $\left\{A_{i}\right\}_{i=1}^{4}$ are mutually exclusive events that jointly span the whole sample space, and hence, they form a partitioned space. Therefore, we can use the total probability theorem to get the distribution of the end-to-end SNR $\Gamma$ :

$$
F_{I S D F}(\gamma)=\sum_{i=1}^{4} F\left(\gamma \mid A_{i}\right) \mathbb{P}\left\{A_{i}\right\}
$$

where

$$
\begin{gathered}
F\left(\gamma \mid A_{1}\right)=\left\{\begin{array}{lc}
\frac{F_{s d}(\gamma)}{\mathcal{P}_{s d}}, & 0<\gamma<\gamma_{t h}, \\
1, & \text { elsewhere }
\end{array}\right. \\
F\left(\gamma \mid A_{3}\right)=F\left(\gamma \mid A_{4}\right)=\left\{\begin{array}{lc}
\frac{F_{s d}(\gamma)-\mathcal{P}_{s d}}{1-\mathcal{P}_{s d}}, & \gamma_{t h}<\gamma<\infty, \\
0, & \text { elsewhere. }
\end{array}\right.
\end{gathered}
$$

Also, we show in Appendix B that

$$
F\left(\gamma \mid A_{2}\right) \approx \begin{cases}F_{1}(\gamma), & 0<\gamma<\gamma_{t h}, \\ F_{2}(\gamma), & \gamma_{t h}<\gamma<\infty,\end{cases}
$$

where $F_{1}(\gamma)$ and $F_{2}(\gamma)$ are given in (31) and (32), respectively, at the top of this page. Substituting (28)-(32) into (27), we get $F_{I S D F}(\gamma)$.

\section{B. $S D F-F D R$}

In SDF-FDR, the relay cooperates regardless of the outage state of the S-D link. Thus, the CDF of the end-to-end SNR can be expressed as:

$$
\begin{aligned}
F_{S D F}(\gamma) & =F\left(\gamma \mid \mathcal{O}_{s r}\right) \mathcal{P}_{s r}+F\left(\gamma \mid \overline{\mathcal{O}}_{s r}\right) \overline{\mathcal{P}}_{s r} \\
& =F_{s d}(\gamma) \mathcal{P}_{s r}+F_{(s, r) \rightarrow d}(\gamma) \overline{\mathcal{P}}_{s r} .
\end{aligned}
$$

By substituting (14), (18) and (22) in (33) we get $F_{S D F}(\gamma)$.

As shown in the last section, both SDF and ISDF yield the same outage performance. However, as shown in this section, SDF has a higher end-to-end SNR profile when compared to ISDF due to the more relay cooperation SDF offers. To motivate their inherent performance difference in light of the above, consider the following illustrative variablerate scenario where the end-to-end communication has $R$ as a lower acceptable rate limit below which an outage is defined and declared. However, when the channel is not in outage with respect to this lower limit, the source is now allowed to freely increase its rate to only approach the verge of outage without falling into it. In this scenario, it is clear that the outage performance will remain the same for both SDF and ISDF since the outage threshold is exactly the same. However, 
it also becomes clear that the protocol which offers a higher end-to-end SNR profile will attain higher rates. Thus, as it will be shown in the subsequent numerical evaluations, ISDF offers additional power savings when compared to SDF while maintaining the same outage performance at the only expense of a one-bit feedback provided that a fixed-rate transmission is adopted. When variable-rate transmission is allowed, assuming channel state information is provided to the transmitters, this power saving can come at an additional expense of a lower end-to-end throughput.

\section{Existing CoOPERAtive Protocols}

\section{A. Assumptions}

Before we proceed with protocol comparisons, we first highlight a few assumptions that have been made.

- We only consider time-orthogonal HDR with nonoverlapping and equal source/relay transmission durations. Non-orthogonal HDR protocols like those in [23] would be still interesting to investigate in the future.

- When comparing settings with different number of transmitters, it is often debatable whether individual or sum transmit power constraints should be imposed, with both approaches being plausible in different scenarios of practical interest. Here, we adopt the individual power constraint scenario which agrees with the distributed nature of cooperative channels in which power budgets cannot be freely exchanged especially when mobility becomes involved, as in uplink communications for instance when the source is a mobile device. This implies that the power of a transmitter is kept fixed regardless of being in a cooperative or non-cooperative scenario.

- When a transmitter experiences intermittent transmission, either power or energy normalization can be adopted. In energy-normalization, a fixed energy budget is allocated for each transmitter regardless of its transmission duration. In agreement with recent studies on the topic [8], we alternatively adopt power-normalization where the energy per time (power) is constrained. This appears more plausible in the sense that radio-frequency (RF) front-ends should stick to the same emission regulations regardless of their transmission durations.

- While comparing FDR to HDR, it is also often questionable how many antennas/RF chains should be allocated for each. In a recent study for multiple-antenna channels [24], two approaches were suggested; namely, antenna conserved versus $R F$ chain conserved. In agreement with recent studies on the topic [8], we adopt herein the RF chain conserved approach where the relay is equipped with the same number of RF chains (one for transmission and another one for reception). This approach neglects the fact that HDR is basically equipped with a single antenna for both transmission and reception as opposed to two antennas in FDR, one for each task, as long as they have the same number of RF chains.

\section{B. Summary of Protocols}

We summarize the protocols considered for comparison in Section VI, taking the direct transmission (DT) as a baseline with rate $R$ bits $/ \mathrm{s} / \mathrm{Hz}$. The protocols are classified under three main categories; namely, 1) multi-hop protocols in which the desired information is only distilled from the multi-hop path while the direct link is neglected/treated as interference, 2) combining protocols where both the multi-hop and direct paths are non-selectively combined, and finally, 3 ) selective relaying protocols in which combining is applied only when the relay performs successful decoding, while the direct link is solely leveraged otherwise. The considered protocols and their endto-end outage probability are summarized in Table I, with $\eta_{1}=$ $2^{R}-1, \eta_{2}=2^{2 R}-1, \eta_{3}=2^{R_{I}}-1$ and $\eta_{4}=2^{R(L+D) / L}-1$. We start by specifying the HDR protocols and their inherent differences in the following progressive way.

a) Multi-hop DF-HDR (MHDF-HDR): Direct source transmissions are totally ignored. An outage is declared unless the S-R and R-D links are simultaneously outage-free, as in Table I.

b) Maximum-Ratio Combining HDR (MRC-HDR) [15]: It allows combining of the direct signal with that arriving via the multi-hop path. Yet, combining might still cause undesirable loss of performance due to the occasional interference from the multi-hop path when an outage occurs in the S$\mathrm{R}$ link. The end-to-end SNR is given in [8], [15] by $\Gamma=$ $\min \left\{\Gamma_{s r}, \Gamma_{r d}+\Gamma_{s d}\right\}$, which can be easily shown to yield the outage probability in Table I.

c) Selective DF-HDR (SDF-HDR) [12], [15]: It overcomes the previous drawback in MRC-HDR by allowing the relay to forward only when the S-R link is not in outage, thereby improving the combining step. However, when the relay does not forward, about half of the time becomes unutilized due to the orthogonal nature of source/relay transmissions. The expression in Table I follows from [15] for Nakagami-m fading and taking power normalization into account.

Due to the rate penalty of $1 / 2$ which all the previous HDR protocols suffer, the transmitters need to signal with a rate of $2 R$ to maintain the same rate adopted by the DT baseline.

d) Incremental DF-HDR (IDF-HDR) [12], [15]: To solve the under-utilization problem in SDF-HDR, the source continues its transmission over the previously dedicated time for relay forwarding as long as the S-D link is not in outage. Hence, IDF-HDR is a hybrid scheme that switches between DT and HDR based on the S-D link outage state. In [15], the source transmits with a constant rate, $R_{I}$, regardless of the S-D link state. The effective rate with rate penalties taken into account is equal to $R_{I} \times 1 \times\left(1-\mathcal{P}_{s d}\right)+R_{I} \times \frac{1}{2} \times \mathcal{P}_{s d}$, with $\mathcal{P}_{s d}$ now calculated for the source rate $R_{I}$. Taking DT as a baseline, this should be equal to $R$. Hence, $R_{I}$ can be calculated by numerically solving the nonlinear equation:

$$
R_{I}:\left(1-F_{X}\left(2^{R_{I}}-1 ; m_{s d}, \theta_{s d}\right) / 2\right) R_{I}=R .
$$

It is clear that $R \leq R_{I} \leq 2 R$ with the lower and upper limits attained at zero and almost sure direct link outage, respectively.

Next, we discuss the FDR protocols. Although FDR versions of the above-listed protocols now exist, their effect on performance is quite different due to two main reasons; the non-orthogonal source/relay transmission in FDR, and the loopback interference link that affects the first hop.

e) Multi-hop DF-FDR (MHDF-FDR) [7]-[10]: The multi- 
TABLE I

A SUMMARY OF THEORETIC OUTAGE PROBABILITY FOR THE DIFFERENT PROTOCOLS UNDER CONSIDERATION.

\begin{tabular}{|c|c|c|c|}
\hline Mode & Protocol & Source Rate & Theoretic Outage Probability \\
\hline & DT & $R$ & $F_{X}\left(\eta_{1} ; m_{s d}, \theta_{s d}\right)$ \\
\hline \multirow{4}{*}{ HDR } & MHDF & $2 R$ & $1-\left(1-F_{X}\left(\eta_{2} ; m_{s r}, \theta_{s r}\right)\right)\left(1-F_{X}\left(\eta_{2} ; m_{r d}, \theta_{r d}\right)\right)$ \\
\hline & MRC & $2 R$ & $1-\left(1-F_{X}\left(\eta_{2} ; m_{s r}, \theta_{s r}\right)\right)\left(1-F_{S}\left(\eta_{2} ; \boldsymbol{p}_{2}\right)\right)$ \\
\hline & SDF & $2 R$ & $F_{X}\left(\eta_{2} ; m_{s r}, \theta_{s r}\right) F_{X}\left(\eta_{2} ; m_{s d}, \theta_{s d}\right)+\left(1-F_{X}\left(\eta_{2} ; m_{s r}, \theta_{s r}\right)\right) F_{S}\left(\eta_{2} ; \boldsymbol{p}_{2}\right)$ \\
\hline & IDF & $R_{I}(34)$ & $F_{X}\left(\eta_{3} ; m_{s r}, \theta_{s r}\right) F_{X}\left(\eta_{3} ; m_{s d}, \theta_{s d}\right)+\left(1-F_{X}\left(\eta_{3} ; m_{s r}, \theta_{s r}\right)\right) F_{S}\left(\eta_{3} ; \boldsymbol{p}_{2}\right)$ \\
\hline \multirow{3}{*}{ FDR } & MHDF & $R$ & $1-\left(1-F_{Z}\left(\eta_{4} ; \boldsymbol{p}_{1}\right)\right)\left(1-F_{Z}\left(\eta_{4} ; \boldsymbol{p}_{2}\right)\right)$ \\
\hline & $\mathrm{BM}$ & $R(L+D) / L$ & {$[13$, Eq. $(5),(7)$, and (11)-(15)] taking $\rho=0.2$} \\
\hline & SDF/ISDF & $R(L+D) / L$ & $F_{Z}\left(\eta_{4} ; \boldsymbol{p}_{1}\right) F_{X}\left(\eta_{4} ; m_{s d}, \theta_{s d}\right)+\left(1-F_{Z}\left(\eta_{4} ; \boldsymbol{p}_{1}\right)\right) F_{S}\left(\eta_{4} ; \boldsymbol{p}_{2}\right)$ \\
\hline
\end{tabular}

hop approach in FDR now imposes undesirable source interference at the destination via the direct link. This comes in addition to the inherent drawback of FDR where a higher outage probability is incurred in the multi-hop path due to the RSI affecting the S-R link. Except for the small delay between source/relay transmissions to maintain causality, MHDF-FDR does not incur a rate penalty in comparison to DT due to its codeword-by-codeword decoding at the destination.

f) Block Markov FDR (BM-FDR) [12], [13]: This protocol was recently applied and analyzed for FDR with RSI in [12], [13] over Rayleigh and Nakagami- $m$ fading channels. Among fixed DF protocols, block Markov superposition encoding [14, and the references therein] attains the best known achievable rates. However, due to its nonselective relaying nature, it falls to some degree under the category of combining protocols which suffer from undesirable loss of performance due to possibly superimposing mere interference from the multi-hop path when S-R link outage occurs. Also, since BM-FDR adopts a block transmission scheme, it incurs a rate penalty of $L /(L+D)$ as in SDF-FDR and ISDF-FDR.

g) Selective/Incremental Selective DF-FDR (SDF$F D R / I S D F-F D R)$ : SDF does not suffer from resource under-utilization anymore since the source almost transmits all the time for large $L / D$, yet its selective nature in the S-R link alleviates the RSI adverse effect on the end-to-end performance. Nonetheless, we modify its rate to $R(L+D) / L$ to account for the rate penalty it incurs due to the delay in block transmission. It is worth mentioning that the simple repetition-based selective cooperation protocols considered herein are only meant to show the effect of selective relaying on the end-to-end performance. Selective cooperation versions of higher rate protocols such as block Markov superposition encoding can be still adopted where it is straightforward to expect their superior performance to their nonselective versions.

\section{Diversity Order}

In Appendix $\mathrm{C}$, we analytically derive and compare the diversity order of SDF-FDR/ISDF-FDR to the widely used MHDF-FDR protocol. It is shown that the proposed selective FDR schemes get around the error floor phenomenon in MHDF-FDR and offers a noticeable diversity gain. It is also shown that with proper loopback isolation/cancellation techniques, the diversity order of FDR can approach that offered by selective HDR protocols. Further insights about all the considered full-duplex and half-duplex schemes are discussed in the following section.

\section{NUMERICAL RESUlts}

\section{A. Simulation Setup}

We generate $10^{7}$ sets of channel realizations according to the channel model described in previous sections for $L=20$ blocks per super-block and $D=2$ blocks, i.e., $k=10$. In each set of channel realizations, we exactly calculate $C_{(s, r) \rightarrow d}$ for SDF/ISDF-FDR as given in (7) without undergoing any approximations. We define the effective end-to-end SNR per symbol in the equivalent single-antenna channel as

$$
\Gamma_{\text {eff }}=2^{\frac{C_{(s, r) \rightarrow d}}{n L}}-1 .
$$

This comes from the equivalence we mentioned earlier between the actual virtual MISO channel and another point-topoint single-antenna channel that can be carefully explained as follows. In the virtual MISO channel, noting that $C_{(s, r) \rightarrow d}$ denotes the exact mutual information per super-block, the mutual information per symbol time can be clearly obtained as $\frac{1}{n(L+D)} C_{(s, r) \rightarrow d}$ since the transmission of $L$ codewords actually spans $L+D$ blocks. On the other hand, a prelog factor of $\frac{n L}{n(L+D)}$ is accordingly introduced in the equivalent singleantenna channel due to the inactivity of the source over the last $D$ blocks, and hence the mutual information per symbol time is given in terms of $\Gamma_{\text {eff }}$ as $\frac{L}{L+D} \log _{2}\left(1+\Gamma_{e f f}\right)$. Thus,

$$
\frac{1}{n(L+D)} C_{(s, r) \rightarrow d}=\frac{n L}{n(L+D)} \log _{2}\left(1+\Gamma_{e f f}\right)
$$

which yields the expression in (35). We compare the empirical probability distribution of this $\Gamma_{\text {eff }}$ with those theoretical results obtained in previous sections. For all the figures we present, we include all used simulation parameters in their caption. We start by evaluating the relative performance of SDF/ISDF-FDR.

\section{B. SDF/ISDF-FDR SNR Performance}

1) $C D F$ : In Fig. 3, we compare the empirical $\mathrm{CDF}$ of the end-to-end SNR with that obtained from the derived expressions for three schemes; namely, (i) DT, (ii) ISDF and (iii) SDF. As depicted in Fig. 3, the CDF of ISDF lies between DT and SDF, and the degree of proximity from either DT or SDF performance is found to depend on the average direct link gain. Specifically, as the direct link gain increases, the ISDF performance gets closer to that of DT due to limited 
cooperation as in Fig. 3(a). This happens for the reason that ISDF does not activate the relay for cooperation as long as the destination can retrieve the source message by solely relying on the direct link. On the other hand, as the direct link gain becomes weaker, the performance of ISDF approaches that of SDF due to the unreliability of the direct link as for instance in Fig. 3(b). This is due to the fact that the destination cannot decode from the source directly, leading to always triggering the relay to cooperate whenever it can decode, which is basically what SDF does. We can also notice that the curves of SDF and ISDF overlap till reaching $\gamma_{t h}=$ $10 \log _{10}\left(2^{R(L+D) / L}-1\right) \mathrm{dB}$. This confirms what was reached earlier in the outage analysis section that both protocols yield the same outage performance. In Fig. 3(a), we kept $m_{s d}=1$ which is reasonable for the direct S-D link, while evaluating the performance for $m_{s r}=m_{r r}=m_{r d}=m \in\{1,3\}$. We can notice that as $m$ increases, the SNR probability distribution shifts to higher values indicating an improvement in performance. However, in contrast to increasing either $m_{s r}$ or $m_{s d}$, which naturally boosts the performance due to decreasing the severity of fading over the multi-hop path, one would expect that increasing $m_{r r}$ should cause a performance degradation due to the introduction of line-of-sight effects to the RSI component. We study this effect in particular in Fig. 3(b) by fixing $m_{s r}=m_{s d}=m_{r d}=1$ and plotting for $m_{r r} \in\{1,4\}$. We found that the effect of increasing $m_{r r}$ for the same set of average channel gains in Fig. 3(a) could not be distinguished. In order to distinguish the said effect, we had to significantly magnify the effect of the RSI channel on the endto-end performance as shown in Fig. 3(b) by simultaneously decreasing the direct link gain to $\pi_{s d}=0 \mathrm{~dB}$ and considerably increasing the RSI link gain to $\pi_{r r}=20 \mathrm{~dB}$. From the shown results, we conclude that the impact of $m_{r r}$ can be negligible as long as $\pi_{r r}$ has a reasonable value that is well below $\pi_{s r}$.

2) Relative Relay Power Expenditure: In Fig. 4, we plot the percentage of relay power expenditure/relay cooperation obtained via simulation for the SDF/ISDF-FDR protocols. We also compare them with their theoretic values of $(1-$ $\left.\mathcal{P}_{s r}\right) \times 100 \%$ and $\left(1-\mathcal{P}_{s r}\right) \mathcal{P}_{s d} \times 100 \%$ for SDF and ISDF, respectively. As observed from the figure, relay power expenditure in SDF steadily decreases with the increase in the attempted source rate. The reason is that, as the source increases its information rate, the relay ability of properly decoding the source message decreases since S-R link outage occurs more frequently. Thus, less cooperation takes place as the rate increases, and hence, the destination gradually proceeds towards relying more on direct source transmissions. On the other hand, ISDF gives a different performance trend, in which the expended relay power increases starting from zero. After some point, it starts its decreasing trend and meets that of SDF until both reach zero power again at high rates. This is due to the fact that, at very low rates, the destination can anyway decode when solely receiving via the direct link. As the rate increases, the direct link starts to fail more frequently, thereby activating the relay cooperation when no outage occurs in the S-R link. As we further increase the rate, outage in the S-R link takes place with higher probability and the relay becomes unable to assist regardless of the direct

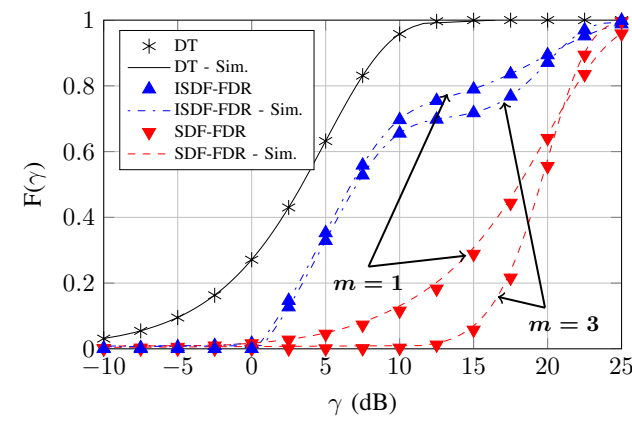

(a) $\pi_{s d}=5 \mathrm{~dB}, \pi_{r d}=\pi_{s r}=20 \mathrm{~dB}, \pi_{r r}=6 \mathrm{~dB}$, $m_{s d}=1, m_{s r}=m_{r r}=m_{r d}=m \in\{1,3\}$ and $R=1 \mathrm{bit} / \mathrm{s} / \mathrm{Hz}$

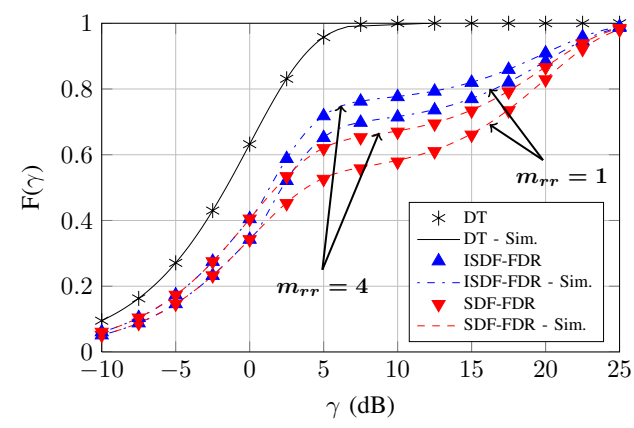

(b) $\pi_{s d}=0 \mathrm{~dB}, \pi_{s r}=\pi_{r r}=\pi_{r d}=20 \mathrm{~dB}, m_{s d}=$ $m_{s r}=m_{r d}=1, m_{r r} \in\{1,4\}$ and $R=1 \mathrm{bit} / \mathrm{s} / \mathrm{Hz}$.

Fig. 3. CDF of the end-to-end SNR.

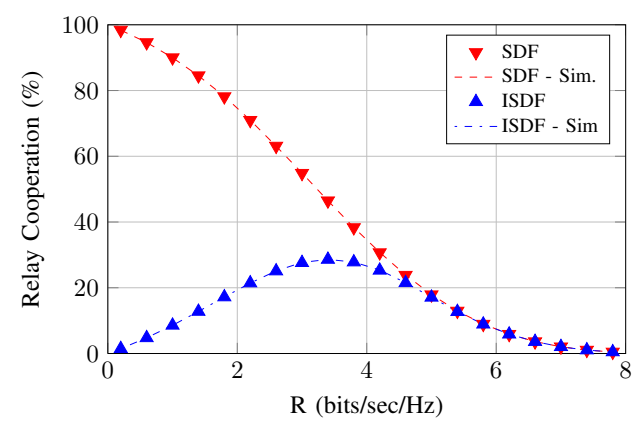

Fig. 4. Percentage of relay cooperation/power expenditure, for $\pi_{s d}=10 \mathrm{~dB}$, $\pi_{s r}=\pi_{r d}=20 \mathrm{~dB}, \pi_{r r}=10 \mathrm{~dB}$ and $m_{s r}=m_{r r}=m_{r d}=m_{s d}=1$.

link's outage state.

In summary, in Fig. 4, there exists at lower rates a nonnegligible probability that the S-D link does not fall into outage, i.e., $1-\mathcal{P}_{s d}$ has a non-negligible value. Hence, relay cooperation is less needed which favors ISDF over SDF since it yields the same outage performance at a lower power consumption. This clearly comes at the expense of a lower SNR profile in the no-outage events for ISDF when compared to SDF, and consequently a lower capacity if a variable rate transmission was allowed. For high rates, the direct link falls into outage with very high probability, i.e., $\mathcal{P}_{s d} \approx 1$. Hence, the relay assists in both protocols whenever it can successfully decode, yielding similar performance for SDF and ISDF as shown. 


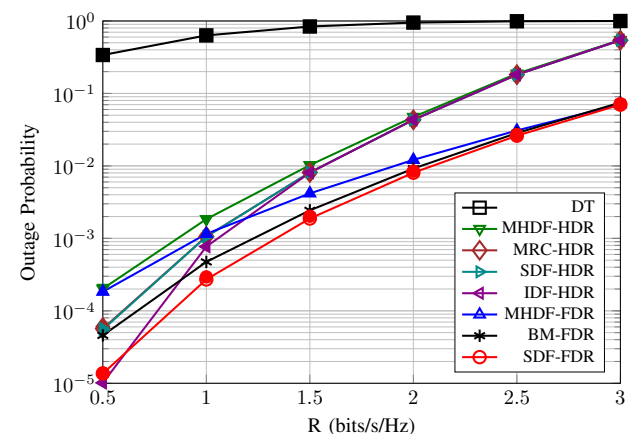

(a) $\pi_{s d}=0 \mathrm{~dB}$

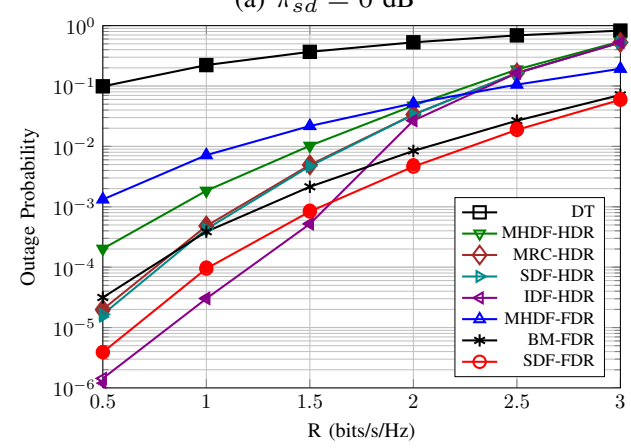

(b) $\pi_{s d}=6 \mathrm{~dB}$

Fig. 5. Outage probability vs. rate, for $\pi_{s r}=\pi_{r d}=20 \mathrm{~dB}, \pi_{r r}=3 \mathrm{~dB}$, $m_{s r}=3, m_{r r}=m_{r d}=2$, and $m_{s d}=1$.

\section{Outage Performance}

In the following figures, we compare the relative outage performance of the FDR and HDR protocols summarized in the previous section. Connected lines with unfilled plot marks of different shapes are dedicated to the theoretic outage probability for each of the protocols, while filled marks of the same respective shape represent the values obtained via simulations. Thus, an appropriately filled mark reflects the quality of matching between theoretical and simulation results. Also, since SDF-FDR and ISDF-FDR yield the same outage performance, we only plot one curve for both to which we refer as SDF-FDR.

1) Outage vs. Spectral Efficiency: In Fig. 5, we plot the outage performance versus the source rate of the DT baseline, denoted by $R$, which also represents the attempted level of spectral efficiency. As shown in Fig. 5(a), SDF-FDR indeed offers better outage performance than all existing schemes for reasonable RSI levels and low direct link gains. As the direct link gain increases, we start to notice in Fig. 5(b) that the performance of IDF-HDR is enhanced. This is due to the fact that its rate/spectral efficiency is significantly enhanced and approaches that of DT as the direct link outage probability decreases, yet it does not suffer from any RSI in the events of direct link outage. Thus, its performance curve shifts down as well as to the right as we increase the direct link gain, and we find that IDF-HDR becomes more desirable for low attempted rates while SDF-FDR remains of better performance for higher rates.

2) Outage vs. Loopback Interference Level: We further clarify the aformentioned observation from a different perspective in Fig. 6, where we plot the outage probability versus

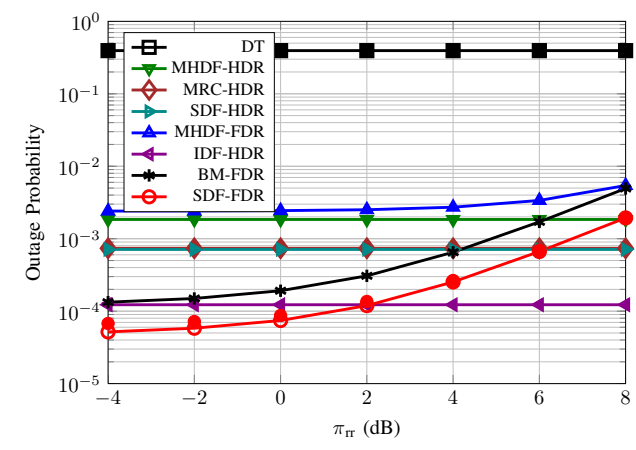

Fig. 6. Outage prob. vs. $\pi_{r r}$, for $\pi_{s r}=\pi_{r d}=20 \mathrm{~dB}, \pi_{s d}=3 \mathrm{~dB}$, $m_{s r}=3, m_{r r}=m_{r d}=2, m_{s d}=1$ and $R=1 \mathrm{bit} / \mathrm{s} / \mathrm{Hz}$.

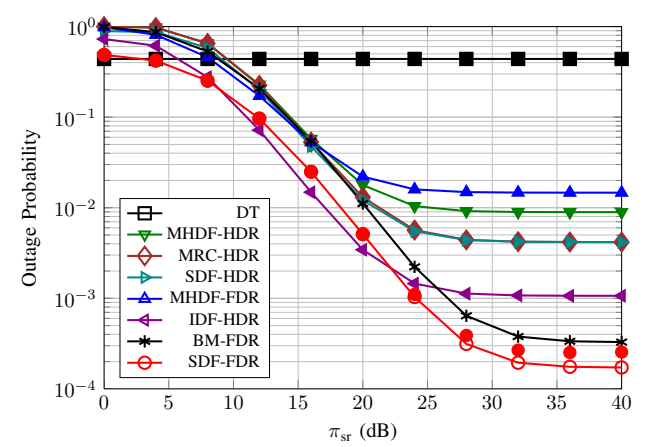

Fig. 7. Outage prob. vs. $\pi_{s r}$, for $\pi_{r d}=20 \mathrm{~dB}, \pi_{r r}=3 \mathrm{~dB}, \pi_{s d}=5 \mathrm{~dB}$, $m_{s r}=m_{r d}=2, m_{r r}=m_{s d}=1$ and $R=1.5 \mathrm{bits} / \mathrm{s} / \mathrm{Hz}$.

$\pi_{r r}$. It can be easily expected that the performance of all HDR protocols remains unchanged as no simultaneous source/relay transmission occurs, while that of FDR protocols suffers gradual deterioration and intersects with the different HDR protocols at different $\pi_{r r}$ levels for any given set of channel parameters. This is obviously caused by the deterioration of the multi-hop path due to the increased level of self-interference.

3) Outage vs. First and Second Hop Gains: In Fig. 7 and Fig. 8, the outage probability is plotted versus $\pi_{s r}$ and $\pi_{r d}$, respectively. The general trend is as shown where the performance is enhanced till reaching the bottleneck of the multi-hop path defined by the other hop, set to $20 \mathrm{~dB}$ in both figures. We noticed however that the relative performance of the incremental/selective HDR and FDR schemes heavily depends on the direct link gain and the targeted spectral efficiency level as previously discussed in Fig. 5. Specifically, the IDF-HDR scheme is significantly improved as the direct link gain increases since its rate $R_{I}$ approaches that of the DT. It is also improved as the the spectral efficiency level, $R$, decreases. On the other hand, the SDF-FDR/ISDF-FDR scheme offer superior performance as the spectral efficiency level decreases.

\section{Diversity Order}

In Fig. 9, we plot the outage probability versus the transmit SNR level at the source and the relay, with both set to $P$, in order to evaluate the diversity order of the different schemes. In Fig. 9(a), we neglect the RSI link for all FDR schemes. As shown, even in the absence of the loopback interference, 


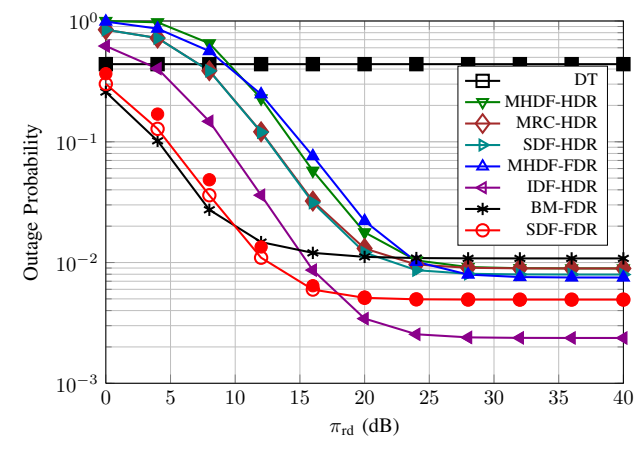

Fig. 8. Outage prob. vs. $\pi_{r d}$, for $\pi_{s r}=20 \mathrm{~dB}, \pi_{r r}=3 \mathrm{~dB}, \pi_{s d}=5 \mathrm{~dB}$, $m_{s r}=m_{r d}=2, m_{r r}=m_{s d}=1$ and $R=1.5 \mathrm{bits} / \mathrm{s} / \mathrm{Hz}$.

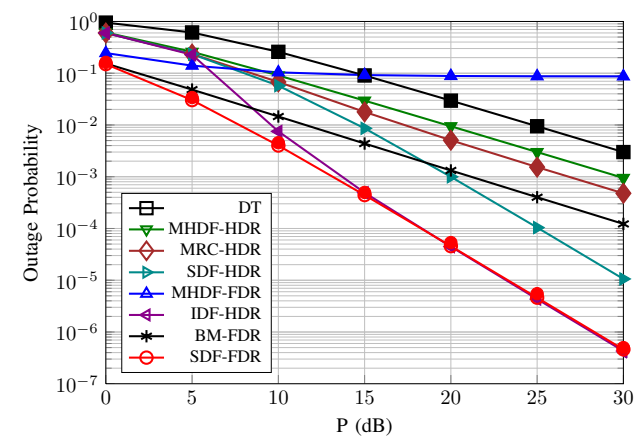

(a) No loopback interference link and large $k$.

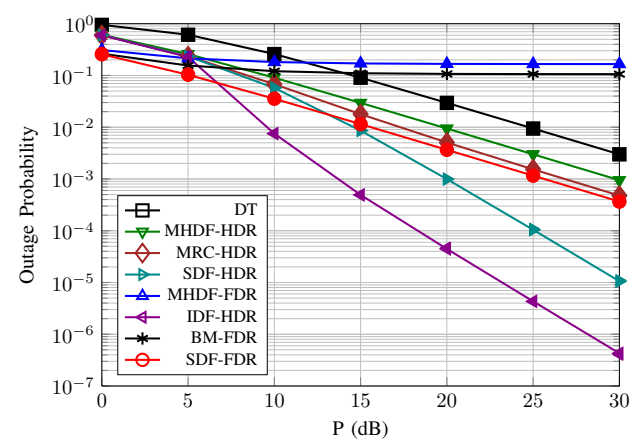

(b) $\pi_{r r}=0 \mathrm{~dB}$, and linearly scaling with $P$.

Fig. 9. Outage prob. vs. $P$, for $\pi_{s r}=\pi_{r d}=15 \mathrm{~dB}, \pi_{s d}=0 \mathrm{~dB}, m_{s r}=$ $m_{r d}=m_{r r}=m_{s d}=1$ and $R=2 \mathrm{bits} / \mathrm{s} / \mathrm{Hz}$.

the MHDF-FDR scheme suffers an error floor as discussed in Appendix $\mathrm{C}$ due to the existence of a nonzero direct link gain. The non-selective HDR schemes, MHDF-HDR and MRCHDR, in addition to the BM-FDR scheme have the same slope of the DT scheme indicating a unity diversity order. This is due to the fact that the three previous schemes have the S$\mathrm{R}$ link as a single-point of failure. When only the S-R link goes into outage, all these three schemes drop into outage as can be noticed from their analytical expressions. On the other hand, the incremental/selective HDR and FDR schemes all enjoy a diversity of order 2 . This agrees with the results from Appendix C, where the multi-hop and direct paths should both fall into outage for an end-to-end outage to occur. It can be seen also that SDF-FDR/ISDF-FDR further outperform the selective/incremental HDR at low-to-moderate (practical) transmit power values.

In Fig. 9(b), we account for the loopback interference link whose gain scales linearly with the relay transmit power. We can notice that diversity order of the full-duplex schemes dropped by 1, i.e., BM-FDR experiences an error floor while the SDF-FDR/ISDF-FDR schemes have a unity diversity order which agrees with the results shown in Appendix C. However, as discussed in the Appendix, the performance of the FDR schemes can be significantly enhanced if the loopback interference isolation and cancellation techniques can control its growth to be rather sublinear with an exponent $0<\delta<1$, i.e., scales with $P^{\delta}$. In this case, the diversity order of the SDFFDR/ISDF-FDR schemes becomes equal to $2-\delta$. Even in this case of linearly scaling loopback interference link, the FDR schemes can still offer better performance than that offered by the HDR schemes at practical transmit power values as shown in previous figures.

\section{E. Discussion}

In the following, we mention some concluding remarks regarding the studied protocols.

- When comparing the outage performance of HDR protocols, we can easily notice as expected that MRC-HDR uniformly dominates MHDF-HDR owing to leveraging the redundant information that arrives via the direct path. Further, SDF-HDR by its turn also uniformly dominates MRC-HDR since it still enjoys the benefits of signal diversity via combining, yet it further eliminates unnecessary interference components from the combining stage when the S-R link falls into an outage state. IDF-HDR offers further performance enhancements by simultaneously eliminating the resource under-utilization in SDFHDR and adjusting its source rate to meet the same level of attempted information rate. Since $R_{I}$ is upper bounded by $2 R$, IDF-HDR replaces $\eta_{2}$ in the outage expression of SDF-HDR by $\eta_{3} \leq \eta_{2}$, and hence, it yields lower outage probability. Indeed, it is worth mentioning that besides the rate adjustment which IDF-HDR offers, it simultaneously captures the gain of selective relaying in SDF-HDR too. This is due to the fact that, due to the incremental nature of IDF-HDR, an event where the multi-hop path disturbs a possibly outage-free direct path can never occur.

- On the other hand, FDR protocols demand about half the source rate of their HDR counterparts to communicate the same amount of information, which should yield a lower outage probability in ideal conditions. Nonetheless, RSI jumps into the picture as an additional challenge that draws the S-R link, and consequently the multi-hop path, into outage more frequently than its HDR counterpart. Thus, an efficient FDR protocol is desirable in which two requirements are simultaneously attained, namely, a) mitigation of the multi-hop path adverse effect on the endto-end performance in the event of S-R link outage, and b) leveraging of the channel diversity elsewhere. From the shown results, we can notice that both requirements are not met in MHDF-FDR, only the second is met in BM-FDR regardless of the S-R link state, while both are attained by SDF/ISDF-FDR.

- In comparing HDR and FDR protocols, the additionally introduced RSI level in FDR plays a pivotal role in 
determining which of the two modes might be preferable over the other, which comes in agreement with recent studies [8]. Indeed, in the absence of RSI, SDF/ISDFFDR outperform all existing HDR and FDR protocols in terms of outage for all possible channel parameters. Yet, depending on the level of RSI, the performance of IDF-HDR gradually improves and can outperform all FDR protocols as we increase the direct link gain or by increasing the source power.

\section{CONCLUSION}

Efficient cooperative protocols were studied for the full-duplex relay channel with loopback interference over Nakagami- $m$ fading channels with integer shape parameters. We proposed an incremental selective decode-and-forward protocol that offers additional power savings to the recently proposed selective decode-and-forward full-duplex cooperation, yet yields the same outage performance which we derived in closed-form. To capture their inherent performance difference, we derived closed-form expressions for the end-to-end signal-to-noise ratio cumulative distribution function for the two protocols, and showed their well-matching behavior with the results obtained via simulation. Also, we compared these protocols to their half-duplex relaying counterparts, as well as existing non-selective full-duplex cooperation protocols in the literature. Our results show that the selective full-duplex protocols outperform their non-selective counterparts, as well as all half-duplex protocols only when self-interference is negligible. On the other hand, with finite levels of selfinterference in full-duplex cooperation, there exist regions of spectral efficiency/direct link gain where either full-duplex or half-duplex cooperation might be favored over the other.

\section{APPENDIX A \\ EIGENVALUES OF $\boldsymbol{H}^{H} \boldsymbol{H}$}

Let $\ell=k d, k \in \mathbb{Z}^{+}$, and consider a family of square matrices $\boldsymbol{A}(\alpha ; \beta ; \ell ; d)=\left(\boldsymbol{a}_{1}, \cdots, \boldsymbol{a}_{\ell}\right)$ of the form:

$$
\boldsymbol{A}(\alpha ; \beta ; \ell ; d)=\alpha \boldsymbol{I}_{\ell}+\beta \boldsymbol{B}_{\ell}{ }^{d}+\beta^{*} \boldsymbol{F}_{\ell}{ }^{d},
$$

where $\alpha \in \mathbb{R}^{+}, \beta \in \mathbb{C}$, while $\boldsymbol{B}_{\ell}\left(\boldsymbol{F}_{\ell}\right)$ denotes a square backward (forward) shift matrix of size $\ell$, with ones only on the first subdiagonal (superdiagonal) and zeros elsewhere. Consider the eigenvalue problem:

$$
\boldsymbol{A u}=\lambda \boldsymbol{u} .
$$

It can be noticed that the nonzero elements of $\boldsymbol{A}$ are limited to positions on the form $(i d+j, j), \forall j \in\{1, \cdots, \ell\}, i \in$ $\{-1,0,1\}$, and $1 \leq i d+j \leq \ell$. This makes a column linearly dependent only on its two neighboring $d$-spaced columns and orthogonal on all others. Motivated by this special structure, we can split $\boldsymbol{A}$ as the sum of $d$ matrices with orthogonal column spaces:

$$
\boldsymbol{A}=\sum_{j=1}^{d} \boldsymbol{A}_{j}
$$

where the matrix $\boldsymbol{A}_{j}$ holds only $k$ nonzero columns corresponding to the $k d$-spaced columns of $\boldsymbol{A}$ with shift $j$, i.e., $\left\{\boldsymbol{a}_{(i-1) d+j}\right\}_{i=1}^{k}$, at their respective positions, while the remaining columns are all zeros. Similarly, let us project the

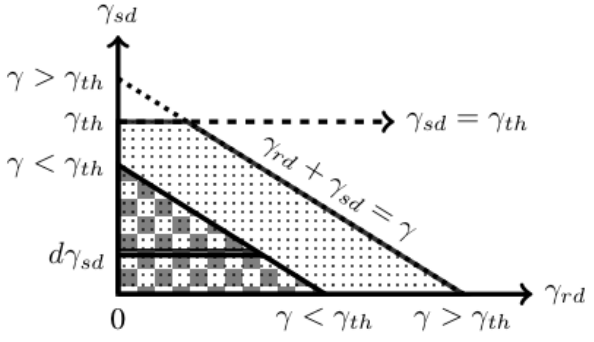

Fig. 10. Integration regions: a) $\gamma<\gamma_{t h}$ (checkerboard), b) $\gamma>\gamma_{t h}$ (dotted).

$\ell \times 1$ eigenvector $\boldsymbol{u}=(u[1], \cdots, u[\ell])^{T}$ onto $d$ orthogonal subspaces, such that:

$$
\boldsymbol{u}=\sum_{j=1}^{d} \boldsymbol{u}_{j}
$$

where the vector $\boldsymbol{u}_{j}$ holds only $k$ nonzero elements corresponding to the $k d$-spaced elements of $\boldsymbol{u}$ with shift $j$, i.e., $\{u[(i-1) d+j]\}_{i=1}^{k}$, at their respective positions, while the remaining elements are all zeros. Thus, the eigenvalue problem can be rewritten as

$$
\sum_{j=1}^{d} \boldsymbol{A}_{j} \sum_{j=1}^{d} \boldsymbol{u}_{j}=\lambda \sum_{j=1}^{d} \boldsymbol{u}_{j},
$$

It is clear that $\boldsymbol{u}_{i}$ lies in the nullspace of $\boldsymbol{A}_{j} \forall i \neq j$. Hence, due to orthogonality, the eigenvalue problem can be split into $d$ eigenvalue problems:

$$
\boldsymbol{A}_{j} \boldsymbol{u}_{j}=\lambda \boldsymbol{u}_{j}, \forall j \in\{1, \cdots, d\} .
$$

Since the $\ell \times \ell \boldsymbol{A}_{j}$ has zero rows and columns corresponding to the zero elements of $\boldsymbol{u}_{j}$, we can eliminate them and alternatively solve the reduced $k$-dimensional eigenvalue problem:

$$
\tilde{\boldsymbol{A}}_{j} \tilde{\boldsymbol{u}}_{j}=\lambda \tilde{\boldsymbol{u}}_{j}, \forall j \in\{1, \cdots, d\},
$$

where $\tilde{\boldsymbol{A}}_{j}$ and $\tilde{\boldsymbol{u}}_{j}$ are $k \times k$ and $k \times 1$, respectively. We can easily notice that $\tilde{\boldsymbol{A}}_{j}=\boldsymbol{A}\left(\alpha ; \beta ; \frac{\ell}{d}, 1\right), \forall j \in\{1, \cdots, d\}$, which is a a tridiagonal Toeplitz matrix with known $k$ eigenvalues as given in (9). Thus, $\boldsymbol{A}(\alpha ; \beta ; \ell, d)$ has the $k$ eigenvalues of $\boldsymbol{A}(\alpha ; \beta ; k, 1)$, each with multiplicity $d$, i.e.,

$$
\lambda_{d(i-1)+1: d i}=\alpha+2|\beta| \cos \frac{i \pi}{k+1}, i \in\{1,2, \cdots, k\} .
$$

Accordingly, its determinant is that of $\boldsymbol{A}(\alpha ; \beta ; k, 1)$ raised to the $d^{t h}$ power. For $n \in \mathbb{Z}^{+}$, substituting with $\ell=n L$ and $d=n D$ in (43) gives the expression in (10).

\section{APPENDIX B \\ DERIVATION OF $F\left(\gamma \mid A_{2}\right)$}

Given $A_{2}, \Gamma_{s d}$ is confined to the range $0<\gamma_{s d}<\gamma_{t h}$, thus having the distribution:

$$
f\left(\gamma_{s d} \mid A_{2}\right)=\left\{\begin{array}{lr}
\frac{f_{s d}\left(\gamma_{s d}\right)}{\mathcal{P}_{s d}}, & 0<\gamma_{s d}<\gamma_{t h}, \\
0, & \text { elsewhere, }
\end{array}\right.
$$

while $\Gamma_{r d}$ remains as $\mathcal{G}\left(m_{r d}, \theta_{r d}\right) \mathrm{RV}$ due to independence. Thus, with the regions of integration depicted in Fig. 10, the $\mathrm{CDF}$ of $\gamma$ conditioned on $A_{2}$ is given by:

$$
\begin{aligned}
F\left(\gamma \mid A_{2}\right) & \approx \mathbb{P}\left\{\Gamma_{s d}+\Gamma_{r d}<\gamma \mid A_{2}\right\} \\
& = \begin{cases}F_{1}(\gamma), & 0<\gamma<\gamma_{t h}, \\
F_{2}(\gamma), & \gamma_{t h} \leq \gamma<\infty,\end{cases}
\end{aligned}
$$


where

$$
\begin{aligned}
& F_{1}(\gamma)=\int_{\gamma_{s d}=0}^{\gamma} f\left(\gamma_{s d} \mid A_{2}\right) F_{r d}\left(\gamma-\gamma_{s d}\right) d \gamma_{s d}, \\
& F_{2}(\gamma)=\int_{\gamma_{s d}=0}^{\gamma_{t h}} f\left(\gamma_{s d} \mid A_{2}\right) F_{r d}\left(\gamma-\gamma_{s d}\right) d \gamma_{s d} .
\end{aligned}
$$

Substituting (1) and (44) in (46) we get

$$
F_{1}(\gamma)=\int_{\gamma_{s d}=0}^{\gamma} \frac{\gamma_{s d}^{m_{s d}-1} e^{-\frac{\gamma_{s d}}{\theta_{s d}} \gamma\left(m_{r d}, \frac{\gamma-\gamma_{s d}}{\theta_{r d}}\right)}}{\mathcal{P}_{s d} \theta_{s d} m_{s d} \Gamma\left(m_{s d}\right) \Gamma\left(m_{r d}\right)} d \gamma_{s d}
$$

For integer $m_{r d}$, we can use the following series expansion of the lower incomplete Gamma function [25, Eq. 8.352-1]:

$$
\frac{\gamma\left(m_{r d}, \frac{\gamma-\gamma_{s d}}{\theta_{r d}}\right)}{\Gamma\left(m_{r d}\right)}=1-e^{-\frac{\gamma-\gamma_{s d}}{\theta_{r d}}} \sum_{m=0}^{m_{r d}-1} \frac{\left(\frac{\gamma-\gamma_{s d}}{\theta_{r d}}\right)^{m}}{\Gamma(m+1)} .
$$

Substituting (49) in (48), and by the Riemann-Liouville integral form in [25, Eq. 3.383-1] we get

where

$$
F_{1}(\gamma)=\frac{I_{11}-I_{12}}{\mathcal{P}_{s d} \theta_{s d} m_{s d} \Gamma\left(m_{s d}\right)},
$$

$$
\begin{aligned}
I_{11} & =\int_{\gamma_{s d}=0}^{\gamma} \gamma_{s d}^{m_{s d}-1} e^{-\frac{\gamma_{s d}}{\theta_{s d}}} d \gamma_{s d} \\
& =\mathcal{B}\left(1, m_{s d}\right) \gamma^{m_{s d}{ }_{1} F_{1}}\left(m_{s d} ; m_{s d}+1 ;-\frac{\gamma}{\theta_{s d}}\right)
\end{aligned}
$$

and

$$
\begin{aligned}
I_{12}= & \int_{\gamma_{s d}=0}^{\gamma} \gamma_{s d}^{m_{s d}-1} e^{-\frac{\gamma_{s d}}{\theta_{s d}}} e^{-\frac{\gamma-\gamma_{s d}}{\theta_{r d}}} \sum_{m=0}^{m_{r d}-1} \frac{\left(\frac{\gamma-\gamma_{s d}}{\theta_{r d}}\right)^{m}}{\Gamma(m+1)} d \gamma_{s d} \\
= & \sum_{m=0}^{m_{r d}-1} \frac{e^{-\frac{\gamma}{\theta_{r d}}}}{\Gamma(m+1) \theta_{r d}} \int_{\gamma_{s d}=0}^{\gamma} \gamma_{s d}^{m_{s d}-1}\left(\gamma-\gamma_{s d}\right)^{m} e^{\frac{\theta_{s d}-\theta_{r d}}{\theta_{s d} \theta_{r d}} \gamma_{s d}} d \gamma_{s d} \\
= & \sum_{m=0}^{m_{r d}-1} \frac{e^{-\frac{\gamma}{\theta_{r d}}} \mathcal{B}\left(m+1, m_{s d}\right) \gamma^{m_{s d}}+m}{\Gamma(m+1) \theta_{r d}{ }^{m}} \\
& \times{ }_{1} F_{1}\left(m_{s d} ; m_{s d}+m+1 ;\left(\frac{1}{\theta_{r d}}-\frac{1}{\theta_{s d}}\right) \gamma\right)
\end{aligned}
$$

with

$$
\mathcal{B}(x, y)=\frac{\Gamma(x) \Gamma(y)}{\Gamma(x+y)}
$$

denoting the beta function [25, Eq. 8.384-1]. Substituting (51) and (52) in (50) we obtain (31). Similarly,

where

$$
F_{2}(\gamma)=\frac{I_{21}-I_{22}}{\mathcal{P}_{s d} \theta_{s d} m_{s d} \Gamma\left(m_{s d}\right)},
$$

$$
I_{21}=\mathcal{B}\left(1, m_{s d}\right) \gamma_{t h}^{m_{s d}}{ }_{1} F_{1}\left(m_{s d} ; m_{s d}+1 ;-\frac{\gamma_{t h}}{\theta_{s d}}\right)
$$

and

$$
\begin{aligned}
I_{22}= & \sum_{m=0}^{m_{r d}-1} \int_{\gamma_{s d}=0}^{\gamma_{t h}} \frac{e^{-\frac{\gamma}{\theta_{r d}}} \gamma_{s d}^{m_{s d}-1}\left(\gamma_{t h}-\gamma_{s d}+\gamma-\gamma_{t h}\right)^{m} e^{\frac{\theta_{s d}-\theta_{r d}}{\theta_{s d} \theta_{r d}} \gamma_{s d}}}{\Gamma(m+1) \theta_{r d}} d \gamma_{s d} \\
= & \sum_{m=0}^{m_{r d}-1} \sum_{k=0}^{m} \frac{\left(\begin{array}{l}
m \\
k
\end{array}\right)\left(\gamma-\gamma_{t h}\right)^{m-k} e^{-\frac{\gamma}{\theta_{r d}}} \mathcal{B}\left(k+1, m_{s d}\right) \gamma_{t h}^{m_{s d}+k}}{\Gamma(m+1) \theta_{r d} d^{m}} \\
& \times{ }_{1} F_{1}\left(m_{s d} ; m_{s d}+k+1 ;\left(\frac{1}{\theta_{r d}}-\frac{1}{\theta_{s d}}\right) \gamma_{t h}\right)
\end{aligned}
$$

In (56), we replaced $\left(\gamma-\gamma_{s d}\right)$ by $\left[\left(\gamma-\gamma_{t h}\right)+\left(\gamma_{t h}-\gamma_{s d}\right)\right]$ and then used the binomial theorem to put the integral on the Riemann-Liouville form in [25, Eq. 3.383-1]. With the substitution of (55) and (56) in (54) and after some manipulations, we get (32). In the first term of (31) and (32), we used the relation between the confluent hypergeometric function and the lower incomplete gamma function in [20, Eq. 6.5.12]. Also, in (32) we used [20, Eq. 13.1.27] to put it on the form in (11).

\section{APPENDIX C \\ DIVERSITY ORDER ANALYSIS}

\section{A. $M H D F$}

We assume Rayleigh-fading links for ease of notation, i.e., $m_{i j}=1$ for $i \in\{s, r\}$ and $j \in\{r, d\}$. By explicitly accounting for the transmit power of the source and the relay with both equal to $P$, the end-to-end outage probability is given from Table I by

$$
\begin{aligned}
\mathcal{P}_{M H} & =1-\left(\frac{\exp \left(-\frac{2^{R}-1}{\pi_{s r} P}\right)}{1+\frac{2^{R}-1}{\pi_{s r}} \pi_{r r}}\right)\left(\frac{\exp \left(-\frac{2^{R}-1}{\pi_{r d} P}\right)}{1+\frac{2^{R}-1}{\pi_{r d}} \pi_{s d}}\right) \\
& =1-\alpha_{1} \exp \left(-\frac{z_{1}}{P}\right),
\end{aligned}
$$

where $z_{i j}=\frac{2^{R}-1}{\pi_{i j}}, z_{1}=z_{s r}+z_{r d}$, and $\alpha_{1}=$ $\frac{1}{\left(1+z_{s r} \pi_{r r}\right)\left(1+z_{r d} \pi_{s d}\right)}$. It is clear that $0<\alpha_{1} \leq 1$ due to the nonnegativity of the channel variances, with $\alpha_{1}=1$ only if $\pi_{r r}=\pi_{s d}=0$. The diversity order [15] is then given by

$$
\begin{aligned}
D_{M H} & =\lim _{P \rightarrow \infty}-\frac{\log \left(\mathcal{P}_{M H}\right)}{\log (P)}, \\
& =\lim _{P \rightarrow \infty}-\frac{\log \left(1-\alpha_{1} \exp \left(-\frac{z_{1}}{P}\right)\right)}{\log (P)} .
\end{aligned}
$$

We can well-approximate $1-\exp \left(-\frac{z_{1}}{P}\right)$ by $\frac{z_{1}}{P}$ as $P$ increases, as in [15]. Thus, for $\alpha_{1}=1$,

$$
D_{M H}=\lim _{P \rightarrow \infty} \frac{\log (P)-\log \left(z_{1}\right)}{\log (P)}=1 .
$$

For $\alpha_{1} \neq 1$, and also for large $P$, we can write $\mathcal{P}_{M H}$ using the aformentioned approximation as

$$
\begin{aligned}
1-\alpha_{1} \exp \left(-\frac{z_{1}}{P}\right) & =1-\alpha_{1}+\alpha_{1}\left(1-\exp \left(-\frac{z_{1}}{P}\right)\right) \\
& \approx\left(1-\alpha_{1}\right)\left(1+\frac{\alpha_{1}}{1-\alpha_{1}} \frac{z_{1}}{P}\right) .
\end{aligned}
$$

Thus, we get

$$
D_{M H}=\lim _{P \rightarrow \infty} \frac{\log \frac{1}{1-\alpha_{1}}-\log \left(1+\frac{\alpha_{1}}{1-\alpha_{1}} \frac{z_{1}}{P}\right)}{\log (P)} .
$$

Taking the limit as $P \rightarrow \infty$, the second term in the numerator goes to $\log (1)=0$ for $\alpha_{1} \neq 1$, while $\log (P)$ goes to $\infty$. Hence, the diversity order $D_{M H}=0$ for $0<\alpha_{1}<1$. It could have been directly observed earlier from (58) that there exists an error floor of $1-\alpha_{1}$ for $\alpha_{1} \neq 1$ as $P \rightarrow \infty$, and thus we readily get $D_{M H}=0$ for $0<\alpha_{1}<1$. This error floor result agrees with recent results in the amplify-and-forward FDR literature [26]. Therefore,

$$
D_{M H}= \begin{cases}0, & \text { if } 0<\alpha_{1}<1, \\ 1, & \text { if } \alpha_{1}=1 .\end{cases}
$$

Hence, for any nonzero value of $\pi_{s d}$ or $\pi_{r r}$, the diversity drops to zero in MHDF. 


\section{B. SDF/ISDF}

In SDF, and under Rayleigh fading, the outage probability is approximately given in [16] by

$$
\begin{aligned}
\mathcal{P}_{S D F} & \approx 1-\exp \left(-\frac{z_{s d}}{P}\right)-\frac{\exp \left(-\frac{z_{s r}}{P}\right)}{\left(1+z_{s r} \pi_{r r}\right)} \overbrace{\frac{\left(\exp \left(-\frac{z_{r d}}{P}\right)-\exp \left(-\frac{z_{s d}}{P}\right)\right)}{\left(1-\frac{\pi_{s d}}{\pi_{r d}}\right)}}^{\mathcal{A}(P)} \\
& =1-\exp \left(-\frac{z_{s d}}{P}\right)-\alpha_{2} \exp \left(-\frac{z_{s r}}{P}\right) \mathcal{A}(P),
\end{aligned}
$$

where $\alpha_{2}=\frac{1}{1+z_{s r} \pi_{r r}}$. It can be easily shown that $\mathcal{A}(P)$ is always positive since the numerator and denominator change their sign simultaneously around the point $\pi_{s d}=\pi_{r d}$. Also, $0<\alpha_{2} \leq 1$, with $\alpha_{2}=1$ only if $\pi_{r r}=0$, i.e., no selfinterference at the full-duplex relay. It can be noticed that $1-\exp \left(-\frac{z_{s d}}{P}\right)$ and $\mathcal{A}(P)$ vanish as $P$ increases for nonzero direct link gain regardless of $\alpha_{2}$, leading to a vanishing $\mathcal{P}_{S D F}$ as $P \rightarrow \infty$. Thus, it is guaranteed that SDF has a nonzero diversity order, which gets around the error floor phenomenon in MHDF even in the presence of self-interference. Now, we quantify the exact diversity order of the SDF scheme. As $P$ increases, we can well-approximate $1-\exp \left(-\frac{z}{P}\right)$ as $\frac{z}{P}$. Hence,

$$
\begin{aligned}
D_{S D F} & \approx \lim _{P \rightarrow \infty}-\frac{\log \left(\frac{z_{s d}}{P}-\alpha_{2} \exp \left(-\frac{z_{s r}}{P}\right) \frac{\frac{z_{s d}}{P}-\frac{z_{r d}}{P}}{1-\frac{\pi_{s d}}{\pi_{r d}}}\right)}{\log (P)} \\
& =\lim _{P \rightarrow \infty}-\frac{\log \left(\frac{z_{s d}}{P}\left(1-\alpha_{2} \exp \left(-\frac{z_{s r}}{P}\right)\right)\right)}{\log (P)},
\end{aligned}
$$

where $\mathcal{A}(P) \approx \frac{\frac{z_{s d}}{P}-\frac{z_{r d}}{P}}{1-\frac{\pi_{s d}}{\pi_{r d}}}=\frac{z_{s d}}{P}$. Hence,

$$
D_{S D F}=1+\lim _{P \rightarrow \infty}-\frac{\log \left(1-\alpha_{2} \exp \left(-\frac{z_{s r}}{P}\right)\right)}{\log (P)} .
$$

The second term in (65) can be obtained similar to the analysis for the MHDF scheme. Specifically, it is equal to zero when $\alpha_{2} \neq 1$, and equal to 1 when $\alpha_{2}=1$, i.e., $\pi_{r r}=0$. Therefore,

$$
D_{S D F}= \begin{cases}1, & \text { if } 0<\alpha_{2}<1 \\ 2, & \text { if } \alpha_{2}=1\end{cases}
$$

It is thereby shown that the SDF scheme can at least maintain a unity diversity order even in the presence of a self-interference link with a gain that scales linearly with the relay power, while it can achieve a diversity of order 2 when the self-interference vanishes (or has a constant value that does not grow with the relay power). In general, if we assume that the RSI link scales sublinearly with the relay power as $P^{\delta} \pi_{r r}$ instead of $P \pi_{r r}$ with $0 \leq \delta \leq 1$, it can be verified using the same previous procedure that the diversity order is equal to $2-\delta$. Hence, the diversity heavily depends on the quality of the adopted loopback isolation and cancellation techniques.

\section{REFERENCES}

[1] E. C. Van Der Meulen, "Three-terminal communication channels," Advances in Applied Probability, pp. 120-154, 1971.

[2] T. Cover and A. El Gamal, "Capacity theorems for the relay channel," IEEE Trans. Inf. Theory, vol. 25, no. 5, pp. 572-584, Sept. 1979.

[3] $\mathrm{H}$. Ju, E. Oh, and D. Hong, "Catching resource-devouring worms in next-generation wireless relay systems: Two-way relay and full-duplex relay," IEEE Commun. Mag., vol. 47, no. 9, pp. 58-65, Sept. 2009.

[4] M. Duarte, C. Dick, and A. Sabharwal, "Experiment-driven characterization of full-duplex wireless systems," IEEE Trans. Wireless Commun., vol. 11, no. 12, pp. 4296-4307, Dec. 2012.
[5] M. Jain, J. Choi, T. Kim, D. Bharadia, S. Seth, K. Srinivasan, P. Levis, S. Katti, and P. Sinha, "Practical, real-time, full duplex wireless," in Proc. ACM MobiCom'11, Las Vegas, NV, Sept. 2011.

[6] M. Duarte and A. Sabharwal, "Full-duplex wireless communications using off-the-shelf radios: Feasibility and first results," in Proc. ASILOMAR'10, Pacific Grove, CA, Nov. 2010.

[7] T. Riihonen, S. Werner, R. Wichman, and J. Hamalainen, "Outage probabilities in infrastructure-based single-frequency relay links," in Proc. IEEE WCNC'09, Budapest, Hungary, Apr. 2009.

[8] T. Riihonen, S. Werner, and R. Wichman, "Hybrid full-duplex/halfduplex relaying with transmit power adaptation," IEEE Trans. Wireless Commun., vol. 10, no. 9, pp. 3074-3085, Sept. 2011.

[9] T. Riihonen, S. Werner, and R. Wichman, "Optimized gain control for single-frequency relaying with loop interference," IEEE Trans. Wireless Commun., vol. 8, no. 6, pp. 2801-2806, June 2009.

[10] P. K. Sharma and P. Garg, "Outage analysis of full duplex decode and forward relaying over Nakagami- $m$ channels," in Communications (NCC), 2013 National Conference on, New Delhi, India, Feb. 2013.

[11] P. K. Sharma and P. Garg, "Performance analysis of full duplex decodeand-forward cooperative relaying over nakagami- $m$ fading channels," Trans. Emerging Tel. Tech., vol. 25, no. 9, pp. 905-913, 2014.

[12] H. Alves, G. Fraidenraich, R. D. Souza, M. Bennis, and M. Latva-aho, "Performance analysis of full duplex and selective and incremental half duplex relaying schemes," in Proc. IEEE WCNC'12, Paris, France, Apr. 2012.

[13] H. Alves, D.B. da Costa, R.D. Souza, and M. Latva-aho, "Performance of block-Markov full duplex relaying with self interference in Nakagami$m$ fading," IEEE Wireless Commun. Lett., vol. 2, no. 3, pp. 311-314, June 2013.

[14] G. Kramer, M. Gastpar, and P. Gupta, "Cooperative strategies and capacity theorems for relay networks," IEEE Trans. Inf. Theory, vol. 51, no. 9, pp. 3037-3063, Sept. 2005.

[15] J.N. Laneman, D.N.C. Tse, and G.W. Wornell, "Cooperative diversity in wireless networks: Efficient protocols and outage behavior," IEEE Trans. Inf. Theory, vol. 50, no. 12, pp. 3062-3080, Dec. 2004.

[16] M. Khafagy, A. Ismail, M.-S. Alouini, and S. Aïssa, "On the outage performance of full-duplex selective decode-and-forward relaying," IEEE Commun. Lett., vol. 17, no. 6, pp. 1180-1183, June 2013.

[17] M. Khafagy, A. Ismail, M.-S. Alouini, and S. Aïssa, "Energy-efficient cooperative protocols for full-duplex relay channels," in Proc. IEEE GLOBECOM'13 Workshops, Atlanta, GA, USA, Dec. 2013.

[18] M. Nakagami, "The m-distribution, a general formula of intensity distribution of rapid fading," Statistical Method of Radio Propagation, 1960.

[19] M. K. Simon and M.-S. Alouini, Digital communication over fading channels, vol. 95, Wiley-Interscience, 2005.

[20] M. Abramowitz and I. A. Stegun, Eds., Handbook of Mathematical Functions, $10^{\text {th }}$ Printing, Dover Publications, Dec. 1972.

[21] W.-C. Yueh and S. S. Cheng, "Explicit eigenvalues and inverses of several toeplitz matrices," The ANZIAM Journal, vol. 48, no. 01, pp. 73-97, July 2006.

[22] A. Chelli and M.-S. Alouini, "On the performance of hybrid-ARQ with incremental redundancy and with code combining over relay channels," IEEE Trans. Wireless Commun., vol. 12, no. 8, pp. 3860-3871, Aug. 2013.

[23] K. Azarian, H. El Gamal, and P. Schniter, "On the achievable diversitymultiplexing tradeoff in half-duplex cooperative channels," IEEE Trans. Inf. Theory, vol. 51, no. 12, pp. $4152-4172$, Dec. 2005.

[24] N. Shende, O. Gurbuz, and E. Erkip, "Half-duplex or full-duplex relaying: A capacity analysis under self-interference," in Proc. CISS'13, Baltimore, MD, USA, Mar. 2013.

[25] I. S. Gradshteyn and I. M. Ryzhik, Table of Integrals, Series, and Products, Seventh Edition, Academic Press, 2007.

[26] I. Krikidis, H. A. Suraweera, P. J. Smith, and C. Yuen, "Full-duplex relay selection for amplify-and-forward cooperative networks," IEEE Trans. Wireless Commun., vol. 11, no. 12, pp. 4381-4393, Dec. 2012. 


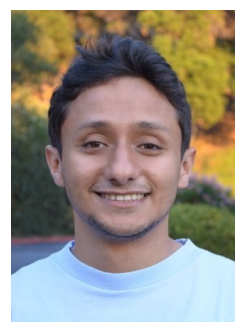

Mohammad Galal Khafagy (S'06, M'15) is a $\mathrm{Ph} . \mathrm{D}$. candidate at King Abdullah University of Science and Technology (KAUST), Thuwal, Makkah Province, Saudi Arabia. He received his B.Sc. degree in Electrical Engineering from the Faculty of Engineering, Alexandria University, Egypt in 2009. He joined the Wireless Intelligent Networks Center (WINC), Nile University, Egypt in 2009 as a graduate research assistant, and he obtained his M.Sc. degree in Wireless Communications from the School of Communication and Information Technology, Nile University, Egypt in 2011. His current research interests include communication theory, information theory, and signal processing for communications.

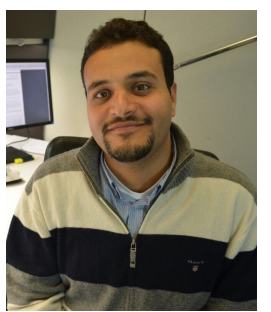

Amr Ismail (S'08 - M'12) was born in Cairo, Egypt in 1982. He received his B.Sc. in 2005 from the Electronics and Communications Engineering (ECE) department, Cairo University, Egypt, and the Diplôme d'ingénieur (M.Sc.) in wireless communications from the École Supérieure d'électricité (SUPELEC), Gif-sur-Yvette, France in 2008. He obtained his Ph.D. degree in telecommunications from SUPELEC in November 2011. He was a postdoctoral fellow at the King Abdullah University of Science and Technology (KAUST), Thuwal, Saudi Arabia till May 2014. Currently, he is a research engineer at Toshiba Research Lab, Bristol, UK. His main research interests include wireless communications and information theory with a special emphasis on multiple-input multipleoutput (MIMO) communications, low-complexity coding/decoding schemes, and interference mitigation techniques.

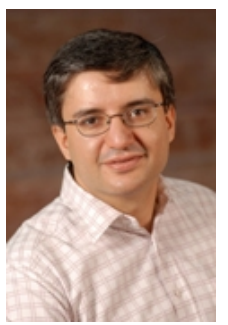

Mohamed-Slim Alouini (S'94, M'98, SM'03, F'09) was born in Tunis, Tunisia. He received the Ph.D. degree in Electrical Engineering from the California Institute of Technology (Caltech), Pasadena, CA, USA, in 1998. He served as a faculty member in the University of Minnesota, Minneapolis, MN, USA, then in the Texas A\&M University at Qatar, Education City, Doha, Qatar before joining King Abdullah University of Science and Technology (KAUST), Thuwal, Makkah Province, Saudi Arabia as a Professor of Electrical Engineering in 2009. His current research interests include the modeling, design, and performance analysis of wireless communication systems.

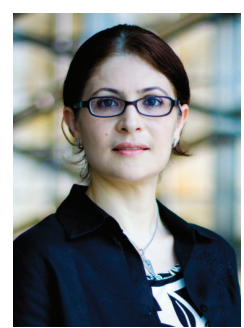

Sonia Aïssa (S'93-M'00-SM'03) received her Ph.D. degree in Electrical and Computer Engineering from McGill University, Montreal, QC, Canada, in 1998. Since then, she has been with the Institut National de la Recherche Scientifique-Energy, Materials and Telecommunications Center (INRS-EMT), University of Quebec, Montreal, QC, Canada, where she is a Full Professor.

From 1996 to 1997, she was a Researcher with the Department of Electronics and Communications of Kyoto University, and with the Wireless Systems Laboratories of NTT, Japan. From 1998 to 2000, she was a Research Associate at INRS-EMT. In 2000-2002, while she was an Assistant Professor, she was a Principal Investigator in the major program of personal and mobile communications of the Canadian Institute for Telecommunications Research, leading research in radio resource management for wireless networks. From 2004 to 2007, she was an Adjunct Professor with Concordia University, Montreal. In 2006, she was Visiting Invited Professor with the Graduate School of Informatics, Kyoto University, Japan. Her research interests lie in the area of wireless and mobile communications, and include radio resource management, cross-layer design and optimization, design and analysis of multiple antenna (MIMO) systems, cognitive and cooperative transmission techniques, performance evaluation, and energy efficiency, with a focus on Cellular and Cognitive Radio networks.

Dr. Aïssa is the Founding Chair of the IEEE Women in Engineering Affinity Group in Montreal, 2004-2007; acted as TPC Leading Chair or Cochair of the Wireless Communications Symposium at IEEE ICC in 2006, 2009, 2011 and 2012; PHY/MAC Program Cochair of the 2007 IEEE WCNC; TPC Cochair of the 2013 IEEE VTC-spring; and TPC Symposia Cochair of the 2014 IEEE Globecom. Her main editorial activities include: Editor, IEEE Transactions ON Wireless CoMmunications, 2004-2012; Associate Editor, IEEE COMMUNICATIONS MAGAZINE, 2004-2009; Technical Editor, IEEE WiRELESS COMMUNICATIONS MAGAZINE, 2006-2010; and Associate Editor, Wiley Security and Communication Networks Journal, 2007-2012. She currently serves as Area Editor for the IEEE TRANSACTIONS ON WIRELESS COMMUNICATIONS, and Technical Editor for the IEEE COMMUNICATIONS MAGAZINE. Awards to her credit include the NSERC University Faculty Award in 1999; the Quebec Government FQRNT Strategic Faculty Fellowship in 2001-2006; the INRS-EMT Performance Award multiple times since 2004, for outstanding achievements in research, teaching and service; and the Technical Community Service Award from the FQRNT Centre for Advanced Systems and Technologies in Communications in 2007. She is co-recipient of five IEEE Best Paper Awards and of the 2012 IEICE Best Paper Award; and recipient of NSERC Discovery Accelerator Supplement Award. She is a Distinguished Lecturer of the IEEE Communications Society (ComSoc) and an Elected Member of the ComSoc Board of Governors. 